\title{
Density response of the warm dense electron gas beyond linear response theory: Excitation of harmonics
}

\author{
Tobias Dornheim $\odot,{ }^{1,2,{ }^{*}}$ Maximilian Böhme $\odot,{ }^{1,2,3}$ Zhandos A. Moldabekov $\odot,{ }^{1,2}$ Jan Vorberger $\odot,{ }^{2}$ and Michael Bonitz $\odot^{4}$ \\ ${ }^{1}$ Center for Advanced Systems Understanding (CASUS), D-02826 Görlitz, Germany \\ ${ }^{2}$ Helmholtz-Zentrum Dresden-Rossendorf (HZDR), D-01328 Dresden, Germany \\ ${ }^{3}$ Technische Universität Dresden, D-01062 Dresden, Germany \\ ${ }^{4}$ Institut für Theoretische Physik und Astrophysik, Christian-Albrechts-Universität zu Kiel, D-24098 Kiel, Germany
}

(Received 7 April 2021; accepted 10 August 2021; published 10 September 2021)

\begin{abstract}
In a recent letter, Dornheim et al. [Phys. Rev. Lett. 125, 085001 (2020)] have investigated the nonlinear density response of the uniform electron gas in the warm dense matter regime. More specifically, they have studied the cubic response function at the first harmonic, which cannot be neglected in many situations of experimental relevance. In this paper, we go one step further and study the full spectrum of excitations at the higher harmonics of the original perturbation based on extensive new ab initio path integral Monte Carlo (PIMC) simulations. We find that the dominant contribution to the density response beyond linear response theory is given by the quadratic response function at the second harmonic in the moderately nonlinear regime. Furthermore, we show that the nonlinear density response is highly sensitive to exchange-correlation effects, which makes it a potentially valuable tool of diagnostics. To this end, we present a theoretical description of the nonlinear electronic density response based on the recent effective static approximation to the local field correction [T. Dornheim et al., Phys. Rev. Lett. 125, 235001 (2020)], which accurately reproduces our PIMC data with negligible computational cost.
\end{abstract}

DOI: 10.1103/PhysRevResearch.3.033231

\section{INTRODUCTION}

Linear response theory (LRT), i.e., the assumption of a linear response of a system of interest to a sufficiently small perturbation, is ubiquitous throughout physics and related disciplines. Prominent examples include the density and current response of many-body systems to external fields, e.g., [1], the computation of optical absorption or plasmon spectra [2,3], the probing of superfluidity [4,5], the description of electronphonon interactions [6], and dynamical simulations based on density functional theory [7]. From a practical point of view, the linear response only depends on the equilibrium properties of the unperturbed system, which often makes an accurate theoretical description feasible in the first place. This also allows one to characterize a system in terms of its response, which is of fundamental importance for diagnostics.

Yet, while it is clear that LRT is only accurate for small perturbations, often the validity of this assumption is not checked. On the one hand, this is quite understandable, as the general nonlinear response is substantially more complicated than LRT and, consequently, much poorer understood. On the other hand, it is well known that nonlinear effects play an important role for many applications, e.g., in the optical excitation and ionization of atoms [8,9], in nonlinear

\footnotetext{
*t.dornheim@hzdr.de

Published by the American Physical Society under the terms of the Creative Commons Attribution 4.0 International license. Further distribution of this work must maintain attribution to the author(s) and the published article's title, journal citation, and DOI.
}

optics [10], in nuclear physics [11], in laser plasmas [12], in the inverse bremsstrahlung heating of plasmas $[13,14]$, nonlinear plasmons in magnetized plasmas [15-17], the excitation spectrum of graphene [18-20], the optical properties of large molecules [21], or intersubband transitions in quantum-well semiconductor heterostructures [22].

The present paper is devoted to the nonlinear density response of warm dense matter (WDM), an exotic state with extreme temperatures $\left(T \sim 10^{4}-10^{8} \mathrm{~K}\right)$ and densities in the vicinity of solid states [23-25]. These conditions occur naturally in astrophysical objects such as giant planet interiors $[26,27]$ and brown dwarfs $[28,29]$. In addition, the compression path of a fuel capsule towards inertial confinement fusion has to traverse the WDM regime [30]. Finally, we mention the potential of hot electrons as a catalyst for chemical reactions [31], and the recent discovery of new materials such as lonsdaleite [32] and nanodiamonds [33] at extreme conditions. Due to this gamut of applications, WDM has emerged as one of the most active frontiers in plasma physics and material science, and WDM is nowadays routinely realized in experiments at large research facilities around the globe; see the recent review article by Falk [34] for an overview of different experimental techniques.

Unfortunately, the theoretical description of WDM is notoriously difficult due to the intriguingly intricate interplay of Coulomb correlations, thermal excitations, and quantum effects. The correlation strength rules out perturbative expansions, e.g. in the frame of Green functions [35], a nonvanishing temperature precludes the applications of the well-stocked arsenal of ground-state methods, and quantum effects make semiclassical methods like molecular dynamics insufficient. Therefore, computationally expensive quantum 
Monte Carlo methods [36,37] have emerged as the method of choice to determine the basic properties of WDM [38-44].

Like theoretical concepts in many other fields, current WDM theory is based, to a large extent, on LRT. For example, the theory of x-ray Thomson scattering $[45,46]$, the most capable diagnostics in WDM experiments, presupposes a purely linear relation between perturbation and response. Other examples include the construction of electronically screened effective potentials [47-49], the estimation of energy-relaxation rates [50-52], and the description of energy-loss properties like the stopping power $[53,54]$. In this context, the central property is often given by the linear density response function $\chi(\mathbf{q}, \omega)$ (with $\mathbf{q}$ and $\omega$ being the wave number and frequency of the perturbation) of the electrons in the system. These can be approximated by the uniform electron gas (UEG) [1], one of the most fundamental model systems in physics and quantum chemistry $[36,55]$.

In a recent letter [56], three of us carried out extensive $a b$ initio path integral Monte Carlo (PIMC) simulations of the harmonically perturbed electron gas in the WDM regime without any assumptions about the response being linear. First and foremost, this has allowed us to check the validity range of LRT in WDM systems. While many applications such as the aforementioned XRTS are safely located within the LRT regime, it was found that it is indeed feasible to induce nonlinear effects using currently available experimental setups. For example, the intentional probing of the nonlinear density response might be developed as an additional method of diagnostics [57]. In addition, we used the exact PIMC data for the density response to extract the cubic density response function at the wave number of the perturbation itself.

In the present paper, we substantially extend these efforts by considering the full excitation spectrum over the entire range of wave numbers. In particular, we find strong nonlinear excitations at the integer higher harmonics of the original perturbation. The quadratic response at the second harmonic constitutes the largest nonlinear contribution for moderate perturbations. Furthermore, we find that the nonlinear response functions strongly depend on electronic exchange-correlation effects, thus making them a potentially valuable new tool of diagnostics. To this end, we introduce a theory of the nonlinear density response based on available representations of the LFC [58,59], which is capable to accurately reproduce our PIMC data with negligible computational cost.

The paper is organized as follows: In Sec. II, we introduce the relevant theoretical background, including the PIMC method (II A), the simulation idea and setup (II B), the extended theory of the nonlinear density response functions (IIC), and the specific way how the latter can be estimated from our PIMC data (IID). In Sec. III, we present our extensive simulation results covering a metallic density (III A), the temperature dependence (III B), and a strongly coupled case (III C). The paper is concluded by a brief summary and outlook in Sec. IV.

\section{THEORY}

We assume Hartree atomic units throughout this work. From a theoretical perspective, the WDM regime is typically defined by two characteristic parameters that are both of the order of one: (i) the density parameter (Wigner-Seitz radius) $r_{s}=\bar{r} / a_{\mathrm{B}}$, where $\bar{r}$ and $a_{\mathrm{B}}$ are the average interparticle distance and first Bohr radius, and (ii) the reduced temperature $\theta=k_{\mathrm{B}} T / E_{\mathrm{F}}$, with $E_{\mathrm{F}}$ being the usual Fermi energy [1]. A third principle parameter is given by the spin-polarization $\xi=$ $\left(N^{\uparrow}-N^{\downarrow}\right) / N$. Throughout this paper, we restrict ourselves to the case of a purely unpolarized system, i.e., $N^{\uparrow}=N^{\downarrow}=N / 2$ and $\xi=0$.

\section{A. Path integral Monte Carlo}

We carry out path integral Monte Carlo (PIMC) simulations $[4,60,61]$ of $N$ electrons in the canonical ensemble, i.e., the volume $V=L^{3}$ (with $L$ being the box length), inverse temperature $\beta=1 / k_{\mathrm{B}} T$, and particle number $N$ are constant.

The basic idea of the PIMC method is to stochastically sample the thermal density matrix

$$
\rho(\mathbf{R}, \mathbf{R}, \beta)=\left\langle\mathbf{R}\left|e^{-\beta \hat{H}}\right| \mathbf{R}\right\rangle,
$$

where $\mathbf{R}=\left(\mathbf{r}_{1}, \ldots, \mathbf{r}_{N}\right)^{T}$ contains the coordinates of all $N$ electrons. In the end, the partition function is recast into the integral over $P$ density matrices at a $P$ times higher temperature, and the resulting high-dimensional integrals are evaluated using the Metropolis Monte Carlo method [62] to avoid the curse of dimensionality of standard quadrature methods; see Ref. [4] for an extensive review article on the PIMC method.

An additional obstacle regarding the PIMC simulations of electrons is the requirement of antisymmetry under particle exchange, which renders the partition function into a sum over both positive and negative contributions. The resulting cancellation of terms with opposite sign is the origin of the notorious fermion sign problem $[63,64]$ that leads to an exponential increase in computation time both with increasing the system size $N$ and with decreasing temperature $T$ [64].

For this reason, one often employs approximate methods like the restricted path integral Monte Carlo method [65] that are not afflicted with the sign problem. Unfortunately, this advantage comes at the cost of an in practice uncontrolled systematic error $[41,43,66]$. Therefore, we here avoid such nodal restrictions and carry out the computationally expensive, yet exact standard PIMC simulations and deal with the sign problem by increasing the computation time, where this is feasible.

For completeness, we mention that all PIMC results presented in this paper have been obtained using a canonical adaption [67] of the worm algorithm by Boninsegni et al. $[68,69]$.

\section{B. Simulation idea}

Following Refs. [56,70-74], we simulate a uniform electron gas that is subject to an external static harmonic perturbation. The corresponding Hamiltonian is given by

$$
\hat{H}=\hat{H}_{\mathrm{UEG}}+2 A \sum_{l=1}^{N} \cos \left(\hat{\mathbf{r}}_{l} \cdot \mathbf{q}\right),
$$

where $\hat{H}_{\text {UEG }}$ is the standard (unperturbed) UEG Hamiltonian $[1,36,55]$. The second term on the right-hand side (r.h.s.) 
of Eq. (2) is the external potential corresponding to a single cosine of wave vector $\mathbf{q}=2 \pi / L\left(n_{x}, n_{y}, n_{z}\right)^{T}$ (with $n_{i} \in \mathbb{Z}$, and $L$ being the length of the simulation box) and the perturbation amplitude $A$. As $A$ corresponds to an energy in this notation, it is given in Hartree throughout this work. Moreover, we restrict ourselves to a strictly static perturbation as PIMC simulations of a time-dependent system are severely hampered by an additional dynamic sign problem [75].

To measure the density response, we compute the induced density

$$
\left\langle\hat{\rho}_{\mathbf{k}}\right\rangle_{q, A}=\frac{1}{V}\left\langle\sum_{l=1}^{N} e^{-i \mathbf{k} \cdot \hat{\mathbf{r}}_{l}}\right\rangle_{q, A},
$$

where $\langle\ldots\rangle_{q, A}$ indicates the parameters of the perturbation in Eq. (2), and $\mathbf{k}$ corresponds to the wave vector at which we measure the response of the system. In particular, within linear response theory it holds

$$
\left\langle\hat{\rho}_{\mathbf{k}}\right\rangle_{q, A}=\delta_{\mathbf{q}, \mathbf{k}} \chi^{(1)}(\mathbf{q}) A,
$$

with $\chi^{(1)}(\mathbf{q})$ being the standard linear response function [1]. The latter is conveniently expressed as

$$
\chi^{(1)}(q)=\frac{\chi_{0}^{(1)}(q)}{1-\frac{4 \pi}{q^{2}}[1-G(q)] \chi_{0}^{(1)}(q)},
$$

where $v(q)=4 \pi / q^{2}$ is the Fourier transform of the Coulomb potential (we use atomic units throughout), $\chi_{0}^{(1)}(q)$ is the density response function of the ideal (non-interacting) Fermi gas that is known from the literature [1], and $G(q)$ is the static local field correction containing the full wave-number resolved information about exchange-correlation effects. In particular, highly accurate data for $G(q)$ have recently become available as a neural-net representation that was constructed on the basis of extensive PIMC simulations $[58,76]$.

In addition, we mention that the full wave-number dependence of $\chi^{(1)}(q)$ can be computed from a single simulation of the unperturbed UEG via the imaginary-time version of the fluctuation-dissipation theorem, which states that

$$
\chi^{(1)}(\mathbf{q})=-n \int_{0}^{\beta} d \tau F(\mathbf{q}, \tau),
$$

with $F(q, \tau)$ being the intermediate scattering function [45] evaluated at an imaginary time argument $\tau \in[0, \beta]$, see Refs. [42,58,77-83] for different applications of this quantity.

Finally, we mention the density of the perturbed electron gas in coordinate space, which, in LRT, is given by

$$
n(\mathbf{r})=n_{0}+2 A \cos (\mathbf{q} \cdot \mathbf{r}) \chi^{(1)}(\mathbf{q}),
$$

with $n_{0}$ being the average value of the density.

\section{Theory of nonlinear density response}

The nonlinear density response of plasmas and condensed matter systems in and out of equilibrium has been studied in some detail theoretically and by simulations, e.g., [84-87]. Here we concentrate on the nonlinear response functions in thermodynamic equilibrium. The general definitions of the second- and third-order response functions are given by an expansion of the induced density, $n_{\text {ind }}(\mathbf{r})=n(\mathbf{r})-n_{0}$, according to a weak perturbation assumption $[88,89]$ :

$$
\begin{aligned}
n_{\text {ind }}(\mathbf{r})= & \int d \mathbf{r}^{\prime} \chi\left(\mathbf{r}, \mathbf{r}^{\prime}\right) V\left(\mathbf{r}^{\prime}\right) \\
& +\int d \mathbf{r}^{\prime} d \mathbf{r}^{\prime \prime} \mathscr{Y}\left(\mathbf{r}, \mathbf{r}^{\prime}, \mathbf{r}^{\prime \prime}\right) V\left(\mathbf{r}^{\prime}\right) V\left(\mathbf{r}^{\prime \prime}\right) \\
& +\int d \mathbf{r}^{\prime} d \mathbf{r}^{\prime \prime} d \mathbf{r}^{\prime \prime \prime} \mathscr{Z}\left(\mathbf{r}, \mathbf{r}^{\prime}, \mathbf{r}^{\prime \prime}, \mathbf{r}^{\prime \prime \prime}\right) V\left(\mathbf{r}^{\prime}\right) V\left(\mathbf{r}^{\prime \prime}\right) V\left(\mathbf{r}^{\prime \prime \prime}\right) \\
& +\cdots,
\end{aligned}
$$

where $V$ is the perturbing potential, whereas $\mathscr{Y}$ and $\mathscr{Z}$ are the second-order and third-order response functions, respectively.

Taking into account that, for a homogeneous system, $\chi\left(\mathbf{r}, \mathbf{r}^{\prime}\right)=\chi\left(\mathbf{r}-\mathbf{r}^{\prime}\right), \mathscr{Y}\left(\mathbf{r}, \mathbf{r}^{\prime}, \mathbf{r}^{\prime \prime}\right)=\mathscr{Y}\left(\mathbf{r}-\mathbf{r}^{\prime}, \mathbf{r}-\mathbf{r}^{\prime \prime}\right)$, and $\mathscr{Z}\left(\mathbf{r}, \mathbf{r}^{\prime}, \mathbf{r}^{\prime \prime}, \mathbf{r}^{\prime \prime \prime}\right)=\mathscr{Z}\left(\mathbf{r}-\mathbf{r}^{\prime}, \mathbf{r}-\mathbf{r}^{\prime \prime}, \mathbf{r}-\mathbf{r}^{\prime \prime \prime}\right)$, Eq. (8) can be rewritten in Fourier space as ( $\Omega$ denotes the volume)

$$
\begin{aligned}
n_{\text {ind }}(\mathbf{k})= & \chi(\mathbf{k}) V(\mathbf{k})+\frac{1}{\Omega} \sum_{\mathbf{k}_{2}} \mathscr{Y}\left(\mathbf{k}-\mathbf{k}_{2}, \mathbf{k}_{2}\right) V\left(\mathbf{k}-\mathbf{k}_{2}\right) V\left(\mathbf{k}_{2}\right) \\
& +\frac{1}{\Omega^{2}} \sum_{\mathbf{k}_{2}} \sum_{\mathbf{k}_{3}} \mathscr{Z}\left(\mathbf{k}-\mathbf{k}_{2}+\mathbf{k}_{3}, \mathbf{k}_{2}, \mathbf{k}_{3}\right) \\
& \times V\left(\mathbf{k}-\mathbf{k}_{2}+\mathbf{k}_{3}\right) V\left(\mathbf{k}_{2}\right) V\left(\mathbf{k}_{3}\right)+\cdots
\end{aligned}
$$

where wave-vector notation $\mathbf{k}$ is used to avoid confusion with the wave vector $\mathbf{q}$ of the external harmonic field $V(\mathbf{r})=$ $2 A \cos \mathbf{q} \cdot \mathbf{r}$.

By substituting into Eq. (9) the Fourier representation of the external potential $(A=\widetilde{A} / \Omega)$,

$$
V(\mathbf{k})=\widetilde{A}\left[\delta_{\mathbf{k}, \mathbf{q}}+\delta_{\mathbf{k},-\mathbf{q}}\right],
$$

we arrive at

$$
\begin{aligned}
n_{\text {ind }}(\mathbf{k})= & \widetilde{A}\left[\chi^{(1)}(\mathbf{k}) \delta_{\mathbf{k}, \mathbf{q}}+\chi^{(1)}(\mathbf{k}) \delta_{\mathbf{k},-\mathbf{q}}\right] \\
& +\frac{\widetilde{A}^{2}}{\Omega}\left[\mathscr{Y}(\mathbf{k}-\mathbf{q}, \mathbf{q}) \delta_{\mathbf{k}, 2 \mathbf{q}}+\mathscr{Y}(\mathbf{k}+\mathbf{q},-\mathbf{q}) \delta_{\mathbf{k},-2 \mathbf{q}}\right] \\
& +\frac{\widetilde{A}^{3}}{\Omega^{2}}\left[\chi^{(1, \text { cubic })}(\mathbf{k}, \mathbf{q}) \delta_{\mathbf{k}, \mathbf{q}}+\chi^{(1, \text { cubic })}(\mathbf{k},-\mathbf{q}) \delta_{\mathbf{k},-\mathbf{q}}\right. \\
& +\mathscr{Z}(\mathbf{k}-2 \mathbf{q}, \mathbf{q}, \mathbf{q}) \delta_{\mathbf{k}, 3 \mathbf{q}} \\
& \left.+\mathscr{Z}(\mathbf{k}+2 \mathbf{q},-\mathbf{q},-\mathbf{q}) \delta_{\mathbf{k},-3 \mathbf{q}}\right] \cdots .
\end{aligned}
$$

The first line contains the linear response $\chi^{(1)}$ at the incoming wave vector $\mathbf{q}$. The second line collects the quadratic response $\mathscr{Y}$ at the second harmonic $2 \mathbf{q}$. The third line gives the cubic response at the first harmonic $\chi^{(1, \text { cubic })}$ where the following notation for brevity was introduced:

$$
\begin{aligned}
\chi^{(1, \text { cubic })}\left(\mathbf{k}, \mathbf{k}^{\prime}\right)= & \mathscr{Z}\left(\mathbf{k}, \mathbf{k}^{\prime},-\mathbf{k}^{\prime}\right)+\mathscr{Z}\left(\mathbf{k},-\mathbf{k}^{\prime}, \mathbf{k}^{\prime}\right) \\
& +\mathscr{Z}\left(\mathbf{k}-2 \mathbf{k}^{\prime}, \mathbf{k}^{\prime}, \mathbf{k}^{\prime}\right) .
\end{aligned}
$$

Finally, the third and fourth lines contain the cubic response function $\mathscr{Z}$ at the third harmonic $3 \mathbf{q}$. We will drop all negative wave vectors from now on as the behavior is symmetric for positive and negative q. It should also be noted that only integer multiples of the original wave vector appear as possible higher-order excitations. 
Now, by performing an inverse Fourier transform of Eq. (11), we obtain, for the induced density in real space,

$$
\begin{aligned}
n_{\text {ind }}(\mathbf{r}) & =\frac{1}{\Omega} \sum_{\mathbf{k}} n_{\text {ind }}(\mathbf{k}) \exp (i \mathbf{k r}) \\
& =2 \sum_{\eta=1}^{\infty}\left\langle\hat{\rho}_{\eta \mathbf{q}}\right\rangle_{q, A} \cos (\eta \mathbf{q} \cdot \mathbf{r})
\end{aligned}
$$

where, for $\eta=1, \eta=2$, and $\eta=3$ :

$$
\begin{gathered}
\left\langle\hat{\rho}_{\mathbf{q}}\right\rangle_{q, A}=\chi^{(1)}(q) A+\chi^{(1, \text { cubic })}(q) A^{3}, \\
\left\langle\hat{\rho}_{\mathbf{2}}\right\rangle_{q, A}=\chi^{(2)}(q) A^{2}, \\
\left\langle\hat{\rho}_{\mathbf{3 q}}\right\rangle_{q, A}=\chi^{(3)}(q) A^{3},
\end{gathered}
$$

and, using Eqs. (11) and (12) we identify

$$
\begin{gathered}
\chi^{(2)}(q)=\mathscr{Y}(\mathbf{k}-\mathbf{q}, \mathbf{q}) \delta_{\mathbf{k}, 2 \mathbf{q}}, \\
\chi^{(1, \text { cubic })}(q)=[\mathscr{Z}(\mathbf{q}, \mathbf{q},-\mathbf{q})+\mathscr{Z}(\mathbf{q},-\mathbf{q}, \mathbf{q}) \\
+\mathscr{Z}(\mathbf{k}-2 \mathbf{q}, \mathbf{q}, \mathbf{q})] \delta_{\mathbf{k}, \mathbf{q}}, \\
\chi^{(3)}(q)=\mathscr{Z}(\mathbf{k}-2 \mathbf{q}, \mathbf{q}, \mathbf{q}) \delta_{\mathbf{k}, 3 \mathbf{q}} .
\end{gathered}
$$

Equations (17) and (19) show the connection between the general second-order $\mathscr{Y}$ and third-order $\mathscr{Z}$ response functions with the response functions of the system at the second and third harmonics $\left[\chi^{(2)}(q)\right.$ and $\chi^{(3)}(q)$, respectively]. Furthermore, Eq. (18) shows the connection of the cubic response at the first harmonic, $\chi^{(1, \text { cubic })}(q)$, to the third-order response function $\mathscr{Z}$.

By definition, the total density in coordinate space $n(\mathbf{r})$ can as well be written as the Fourier series over all wave numbers $\mathbf{q}$,

Yet, as only the harmonics of the perturbation wave vector q exhibit a nonzero response, the density is simply

$$
n(\mathbf{r})=n_{0}+2 \sum_{\eta=1}^{\infty}\left\langle\hat{\rho}_{\eta \mathbf{q}}\right\rangle_{q, A} \cos (\eta \mathbf{q} \cdot \mathbf{r}) .
$$

\section{Noninteracting case}

A general expression for the ideal second-order response function, $\mathscr{Y}_{0}$, and some of its properties were considered in Refs. [88-98].

Mikhailov [19,99] expressed the ideal response functions at the second harmonic $\chi_{0}^{(2)}(q)$, and third harmonic $\chi_{0}^{(3)}(q)$ in terms of the first-order response function $\chi_{0}^{(1)}(q)$ without invoking $\mathscr{Y}_{0}(\mathbf{k}, \mathbf{q})$ and $\mathscr{Z}_{0}\left(\mathbf{k}, \mathbf{k}^{\prime}, \mathbf{q}\right)$ but by directly expanding the induced density at harmonics. The recursion relations derived by Mikhailov [19,99] are

$$
\chi_{0}^{(2)}(q)=\frac{2}{q^{2}}\left(\chi_{0}^{(1)}(2 q)-\chi_{0}^{(1)}(q)\right),
$$

and [19]

$$
\chi_{0}^{(3)}(q)=\frac{3 \chi_{0}^{(1)}(3 q)-8 \chi_{0}^{(1)}(2 q)+5 \chi_{0}^{(1)}(q)}{3 q^{4}} .
$$

Such a recursion formula does not exist for the ideal cubic response at the first harmonic $\chi^{(1, \text { cubic })}(q)$. In fact, the terms making up the ideal cubic response at the first harmonic diverge.

\section{Random phase approximation}

Taking into account screening in linear approximation, the RPA result for the response function from the Green's functions based consideration has the following form [88]:

$$
\mathscr{Y}(\mathbf{k}-\mathbf{q}, \mathbf{q})=\frac{\mathscr{Y}(\mathbf{k}-\mathbf{q}, \mathbf{q})}{\left[1-v(\mathbf{q}) \chi_{0}^{(1)}(\mathbf{q})\right]^{2}\left[1-v(\mathbf{k}) \chi_{0}^{(1)}(\mathbf{k})\right]} .
$$

At the second harmonic $\chi_{\mathrm{RPA}}^{(2)}(q)$ is found using the relation (17) and Eq. (23),

$$
\chi_{\mathrm{RPA}}^{(2)}(q)=\frac{\chi_{0}^{(2)}(q)}{\left[1-v(q) \chi_{0}^{(1)}(q)\right]^{2}\left[1-v(2 q) \chi_{0}^{(1)}(2 q)\right]} .
$$

To illustrate the level of approximation in Eqs. (23) and (24), it is instructive to provide an alternative derivation of Eq. (24). To begin with, we recall that, in contrast to the ideal response function, the response function in RPA takes into account interelectronic interactions in a mean field approximation. Therefore, introducing a total potential as a sum of the external potential and the Hartree potential due to the induced electronic density, $\Phi_{\text {tot }}=\Phi_{\text {ext }}+\Phi_{\text {ind }}$, the induced density in RPA at the second harmonic is obtained in terms of the ideal response functions:

$$
\begin{aligned}
n_{\text {ind }}^{\mathrm{RPA}}(2 q)= & \chi_{0}^{(2)}(q)\left[\Phi_{\text {tot }}(q)\right]^{2}+\chi_{0}^{(1)}(2 q) \Phi_{\text {ind }}(2 q) \\
& +\chi_{0}^{(1, \text { cubic })}(2 q)\left[\Phi_{\text {ind }}(2 q)\right]^{3}
\end{aligned}
$$

where the first term on the r.h.s. of Eq. (25) is the second-order response of the system, which is proportional to the square of the perturbing potential [as can be seen from Eq.(15)], the second and third terms on the r.h.s. of Eq. (25) are contributions from the linear and cubic responses induced by $\Phi_{\text {ind }}$ at $2 q$ [in accordance with Eq. (14)].

On the other hand, the induced density in RPA is expressed as the response to the external field using the quadratic response function in RPA,

$$
n_{\text {ind }}^{\mathrm{RPA}}(2 q)=\chi_{\mathrm{RPA}}^{(2)}(q)\left[\Phi_{\text {ext }}(q)\right]^{2} .
$$

From Eqs. (26) and (25), we find

$$
\begin{aligned}
\chi_{\mathrm{RPA}}^{(2)}(q)\left[\Phi_{\mathrm{ext}}(q)\right]^{2}= & \chi_{0}^{(2)}(q)\left[\Phi_{\mathrm{tot}}(q)\right]^{2}+\chi_{0}^{(1)}(2 q) \Phi_{\text {ind }}(2 q) \\
& +\chi_{0}^{(1, \text { cubic })}(2 q)\left[\Phi_{\text {ind }}(2 q)\right]^{3} .
\end{aligned}
$$

To solve Eq. (27), we combine the Poisson equation for the induced potential at the second harmonic with Eq. (26) and find a relation between $\Phi_{\text {ind }}(2 q)$ and $\Phi_{\text {ext }}(q)$,

$$
\begin{aligned}
\Phi_{\text {ind }}(2 q)=\Phi_{\text {tot }}(2 q) & =\frac{4 \pi}{(2 q)^{2}} n_{\text {ind }}^{\mathrm{RPA}}(2 q) \\
& =\frac{4 \pi}{(2 q)^{2}} \chi_{\mathrm{RPA}}^{(2)}(q)\left[\Phi_{\mathrm{ext}}(q)\right]^{2},
\end{aligned}
$$

where it was taken into account that there is no contribution from the external potential to the total potential at the second harmonic. 
Secondly, we neglect the contribution from the cubic term, i.e., set $\chi_{0}^{(1, \text { cubic })}(2 q)=0$ in Eqs. (25) and (27), and thus approximate the total potential at the first harmonic using linear response theory, which reads in RPA

$$
\Phi_{\mathrm{tot}}(q)=\frac{\Phi_{\mathrm{ext}}(q)}{1-v(q) \chi_{0}^{(1)}(q)} .
$$

Using Eqs. (28) and (29), we can solve Eq. (27) with respect to $\chi_{\mathrm{RPA}}^{(2)}(q)$ to find Eq. $(24)$.

For further discussion of the QMC data, it is important to emphasize that, in $\chi_{\mathrm{RPA}}^{(2)}$ defined by Eq. (24), (i) the screening is taken into account only in linear approximation and (ii) the contribution due to the cubic response function is neglected.

Next, by following the steps from Eq. (25) to Eq. (27), but for the induced density at the third harmonic, and making use of the screening in linear approximation, we find for the response function at the third harmonic in RPA:

$$
\chi_{\mathrm{RPA}}^{(3)}(q)=\frac{\chi_{0}^{(3)}(q)}{\left[1-v(q) \chi_{0}^{(1)}(q)\right]^{3}\left[1-v(3 q) \chi_{0}^{(1)}(3 q)\right]} .
$$

Note that for a weak perturbation the neglected contribution to the screening due to the cubic response $\chi_{0}^{(1, \text { cubic })}$ in Eq. (24) is a higher-order correction compared to the quadratic response. In contrast, for $\chi_{\mathrm{RPA}}^{(3)}$, the neglected cubic response $\chi_{0}^{(1, \text { cubic })}$ has the same order contribution as $\chi_{0}^{(3)}$. Therefore, it can be expected that Eq. (24) performs better than Eq. (30) when applied for the description of the QMC data at the corresponding harmonics.

The corresponding cubic response in RPA at the first harmonic is given by

$$
\chi_{\mathrm{RPA}}^{(1, \text { cubic })}(q)=\frac{\chi_{0}^{(1, \text { cubic })}(q)}{\left[1-v(q) \chi_{0}^{(1)}(q)\right]^{4}} .
$$

It is known that there are further terms that are technically contributing to the cubic response at the level of RPA. These terms are made up from nondiagonal quadratic response functions entirely $[100,101]$.

\section{Going beyond the RPA description by using LFC}

In Eqs. (24) and (30), the electronic interactions effect is included on the basis of the linear response functions in the denominator. Therefore, in analogy to the usual practice in linear response theory, we can go beyond RPA by introducing a local field correction (LFC) $G(q)$. In this way, we arrive at the following equations for the response functions at the second and third harmonics with higher-order electronic exchange-correlations effect included by using LFC:

$$
\begin{aligned}
\chi_{\mathrm{LFC}}^{(2)}(q)= & \chi_{0}^{(2)}(q)\left[1-v(q)[1-G(q)] \chi_{0}^{(1)}(q)\right]^{-2} \\
& \times\left[1-v(2 q)[1-G(2 q)] \chi_{0}^{(1)}(2 q)\right]^{-1}
\end{aligned}
$$

and

$$
\begin{aligned}
\chi_{\mathrm{LFC}}^{(3)}(q)= & \chi_{0}^{(3)}(q)\left[1-v(q)[1-G(q)] \chi_{0}^{(1)}(q)\right]^{-3} \\
& \times\left[1-v(3 q)[1-G(3 q)] \chi_{0}^{(1)}(3 q)\right]^{-1} .
\end{aligned}
$$

Equations (33) and (34) provide an improved description of the response functions on the second and third harmonics with electronic exchange-correlation effects taken into account on the level of the linear response theory. We next give the respective result for the cubic response at the first harmonic,

$$
\chi_{\mathrm{RPA}}^{(1, \text { cubic })}(q)=\frac{\chi_{0}^{(1, \text { cubic })}(q)}{\left[1-v(q)[1-G(q)] \chi_{0}^{(1)}(q)\right]^{4}} .
$$

The temperature- and density-dependent static LFC has been obtained from QMC simulations recently [58,73,74,77,102]. Furthermore, Dornheim et al. [59,76] have introduced the concept of the effective static approximation to the LFC, which is available as a simple analytical representation [59].

\section{PIMC approach to nonlinear density response functions}

To extract the nonlinear density response functions of different harmonics from our PIMC simulation, we evaluate Eq. (3) given above for different perturbation amplitudes $A$ and subsequently perform fits to these data.

In particular, the density response in reciprocal space for the same wave number as the perturbation is fitted to [71,72]

$$
\left\langle\hat{\rho}_{\mathbf{q}}\right\rangle_{q, A}=\chi^{(1)}(q) A+\chi^{(1, \text { cubic })}(q) A^{3},
$$

where the coefficients $\chi^{(1)}(q)$ and $\chi^{(1, \text { cubic })}(q)$ are the free parameters. Note that the determination of $\chi^{(1)}(q)$ from the PIMC data via Eq. (36) is redundant as it is already known from previous simulations of the unperturbed UEG via Eq. (6). However, these independent benchmark data can be compared to our new results for $\chi^{(1)}(q)$ and thus constitute a valuable consistency check of our approach, see also Ref. [56]. Furthermore, it is important to note that Eq. (36) only holds up to a maximum perturbation strength, beyond which contributions with a higher order of $A$ start to substantially contribute; see Sec. III for a hands-on discussion of this point.

The density response of the second harmonic is, in first order in $A$, given by

$$
\left\langle\hat{\rho}_{2 \mathbf{q}}\right\rangle_{q, A}=\chi^{(2)}(q) A^{2},
$$

where $\chi^{(2)}(q)$ is the free parameter that is obtained from a fit to the $A$ dependence for a fixed perturbation wave number $q$. Similarly, the density response of the third harmonic is obtained by fitting the PIMC data for the response at $\mathbf{k}=3 \mathbf{q}$ to

$$
\left\langle\hat{\rho}_{3 \mathbf{q}}\right\rangle_{q, A}=\chi^{(3)}(q) A^{3}
$$

\section{RESULTS}

\section{A. Metallic density: $r_{s}=2$}

Let us start our investigation by considering the response of the electron gas at $r_{s}=2$ and $\theta=1$. These conditions are located at the center of the WDM regime and are realized experimentally for example in experiments with aluminum, e.g., Ref. [103]. In Fig. 1, we show the density response of the system in dependence on the perturbation amplitude $A$ for the same wave number as the perturbation, i.e., for the first harmonic $\left\langle\hat{\rho}_{\mathbf{q}}\right\rangle_{q, A}$. The green crosses show the raw PIMC data, which have been obtained by evaluating Eq. (3). The solid red line shows the prediction by LRT and has been obtained 


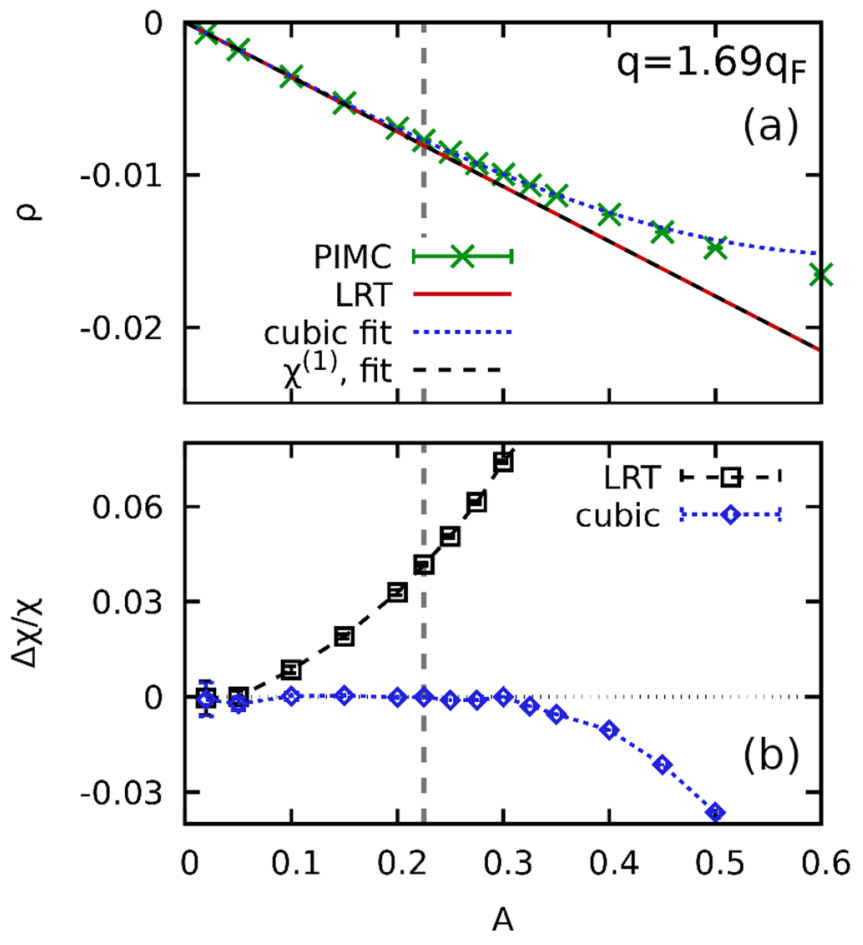

FIG. 1. Panel (a): Dependence of the density response of the first harmonic $\left\langle\hat{\rho}_{\mathbf{q}}\right\rangle_{q, A}$ of the UEG on the perturbation amplitude $A$ for $N=14, r_{s}=2$, and $\theta=1$ with the perturbation wave number $q \approx$ $1.69 q_{\mathrm{F}}$. The green crosses are PIMC data evaluated from Eq. (3), and the solid red line is the prediction from LRT, cf. Eq. (6). The dotted-blue line has been obtained by fitting Eq. (36) to the PIMC data within the interval $A \in[0,0.225]$ (vertical grey dashed line), and the dashed-black line shows the corresponding LRT prediction from the first coefficient only. Panel (b) shows the deviation of LRT and the cubic fit to the PIMC data.

from a previous simulation of the unperturbed UEG [58] by evaluating Eq. (6). Evidently, LRT is in excellent agreement with the PIMC data for small $A$ only and the disagreement rapidly increases with $A$. This can be seen particularly well in Fig. 1(b) showing the relative deviation to the PIMC data. For completeness, we mention that a similar analysis has been presented in Ref. [56], although for a different wave number.

The dotted-blue curve has been obtained by fitting Eq. (36) to the PIMC data within the interval $A \in[0,0.225]$, see also the vertical dashed-grey line. Evidently, this curve captures the emerging deviations between the green crosses and the red line and remains accurate for substantially larger values of $A$. In particular, it remains accurate even beyond the fitting interval, which constitutes a strong empirical confirmation of the functional form in Eq. (36). In addition, the dashed-black line shows the LRT prediction using the first coefficient from the fit and perfectly agrees with the independent red line. Still, we note that the blue curve, too, eventually becomes inaccurate, as the higher-order terms in $A$ that are neglected in Eq. (36) start to significantly contribute.

A possibly more intuitive illustration of the impact of the harmonic perturbation on the system is presented in Fig. 2 where we show the density of the system along the direction of the perturbation. More specifically, the left column corre- sponds to $A=0.02$, which falls well into the LRT regime, cf. Fig. 1. Consequently, the red curve that has been obtained from Eq. (7) is in excellent agreement to the green crosses depicting the PIMC data. The relative deviation of LRT to the latter is shown in the bottom panel (c) of the same figure, and we find perfect agreement within the given Monte Carlo error bars that are of the order of $\Delta n / n \lesssim 0.1 \%$. Overall, we find that the density modulation attains an amplitude of almost $5 \%$ of the unperturbed density.

Let us next turn to the right column of Fig. 2 showing the same quantity for a larger perturbation amplitude, $A=0.2$. In this case, the density modulation is substantially larger and the amplitudes of $n(x)$ are of the order of $50 \%$ with respect to the unperturbed density. Thus, the system is indeed to a large degree shaped by the presence of the external potential. Consequently, the red LRT curve only gives a qualitative description of the density and systematically underestimates (overestimates) the height of the peaks (depth of the minima) of $n$. This can again be seen most clearly in the deviation plot in the bottom panel (d), where we find differences between LRT and the PIMC data exceeding 5\%.

As a first step towards an improved description of the density profile $n(x)$, we go beyond LRT by including the cubic contribution $\chi^{(1, \text { cubic })}$ to the first harmonic. Indeed, Fig. 1 clearly indicates that this provides a fully adequate description of $\left\langle\hat{\rho}_{\mathbf{q}}\right\rangle_{q, A}$, and the dash-dotted black curve in Fig. 2 corresponds to Eq. (20) truncated after $\eta=1$. Let us list a few observations: (i) the impact of $\chi^{(1, \text { cubic })}$ on $n(x)$ is relatively small despite the large density modulation amplitude and the inaccurate description of LRT; (ii) from panel (a) we see that $\chi^{(1, \text { cubic })}$ leads to a somewhat improved agreement with the PIMC data around the minima but increased deviations around the peaks. This becomes more clear by looking at $\Delta n / n_{0}$ in panel (b). The LRT curve exhibits a deviation profile that oscillates twice as fast in space as the original density modulation, but the amplitude of this deviation is not constant. In fact this deviation profile is a combination of (a) the insufficient description of $\left\langle\hat{\rho}_{\mathbf{q}}\right\rangle_{q, A}$ by LRT (cf. Fig. 1) and (b) the omission of the second harmonic that does, by definition, oscillate twice as fast in space as the first one. By including $\chi^{(1, \text { cubic })}$ in the description of $\left\langle\hat{\rho}_{\mathbf{q}}\right\rangle_{q, A}$, we have removed effect (a), and the residual deviation profile (black squares in the bottom panel of Fig. 2) does indeed have a constant amplitude and corresponds to the contribution of the second harmonic.

Evaluating Eq. (20) up to $\eta=2$ gives the dashed blue curve, which is in striking agreement with the green crosses, over the entire $x$ range. This is again confirmed by the deviation plot in the bottom, where the corresponding blue diamonds fluctuate around zero deviation with a relative accuracy of $\Delta n \sim 0.1 \%$.

A more systematic investigation of the emerging impact of higher harmonics upon increasing the perturbation amplitude is presented in Fig. 3, where we show the full wave-number dependence of $\left\langle\hat{\rho}_{\mathbf{k}}\right\rangle_{q, A}$ for different values of $A$. Panel (a) shows results for $q \approx 1.69 q_{\mathrm{F}}$, i.e., the same perturbation wave number as in Figs. 1 and 2. First and foremost, we empirically confirm that a nonzero value for the density response can indeed only be found at the integer harmonics of the original perturbation wave vector q. For the smallest depicted value 

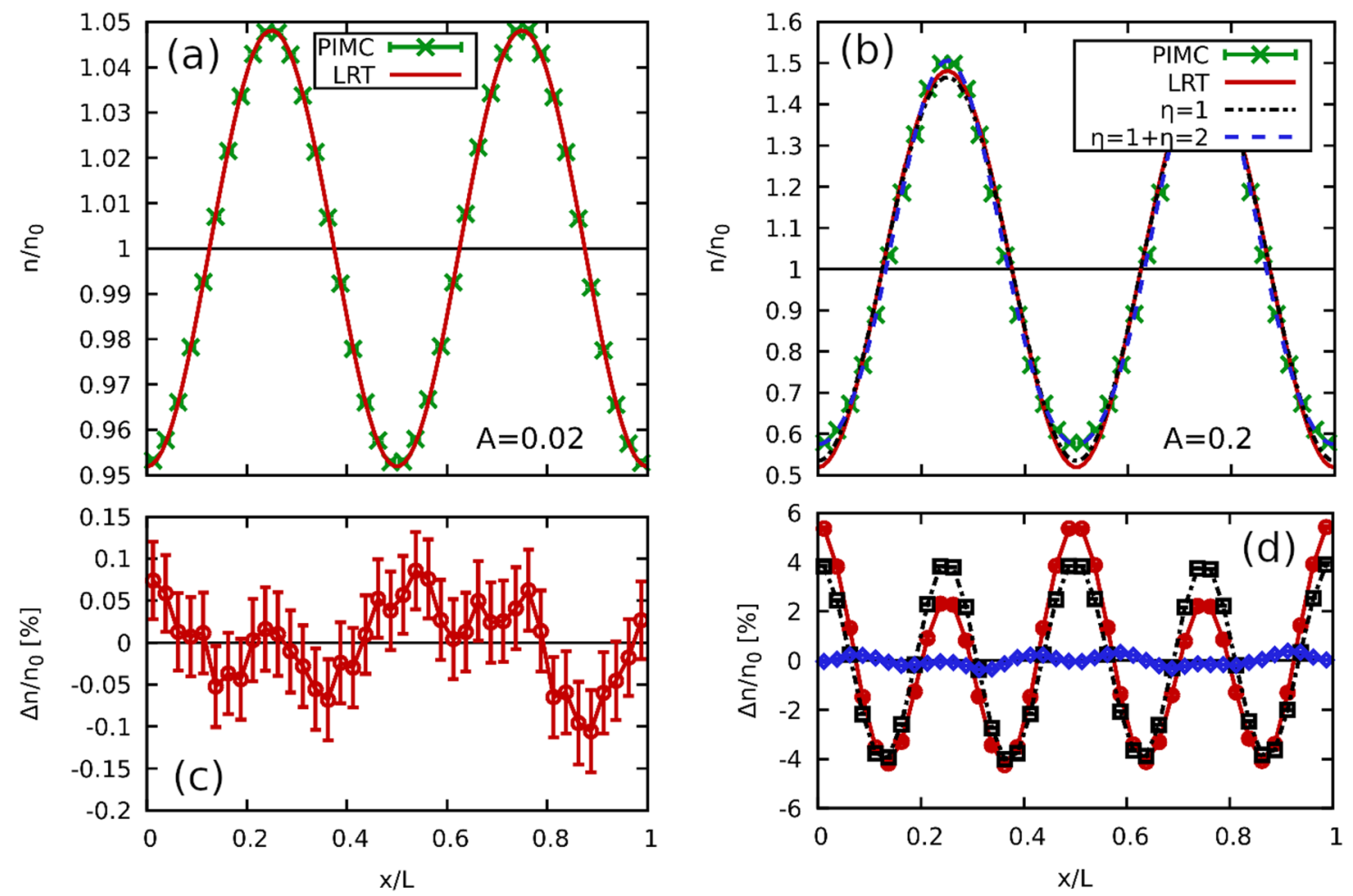

FIG. 2. Density profile of the harmonically perturbed electron gas for $N=14, r_{s}=2$, and $\theta=1$ with $q \approx 1.69 q_{\mathrm{F}}$ and $\mathbf{q}=2 \pi / L(2,0,0)^{T}$ for $A=0.02$ (a) and $A=0.2$ (b). Top row: Density $n(x)$ along the direction of the perturbation, with the green crosses and solid-red curves corresponding to PIMC data and LRT [cf. Eq. (7)]. The dash-dotted black and dashed-blue curves have been obtained by truncating Eq. (20) after the first $(\eta=1)$ and second $(\eta=2)$ harmonic, respectively. The bottom panels (c) and (d) show the corresponding deviation of different theories to the PIMC data.

of the perturbation amplitude, $A=0.02$ (black squares), we only find a signal at $q$ itself, which agrees with the prediction known from LRT. Increasing the perturbation strength by a factor of five $(A=0.1)$ leads to the results given by the red circles. For the first harmonic, the response remains almost unchanged and varies only by $\sim 1 \%$ (cf. the bottom panel of

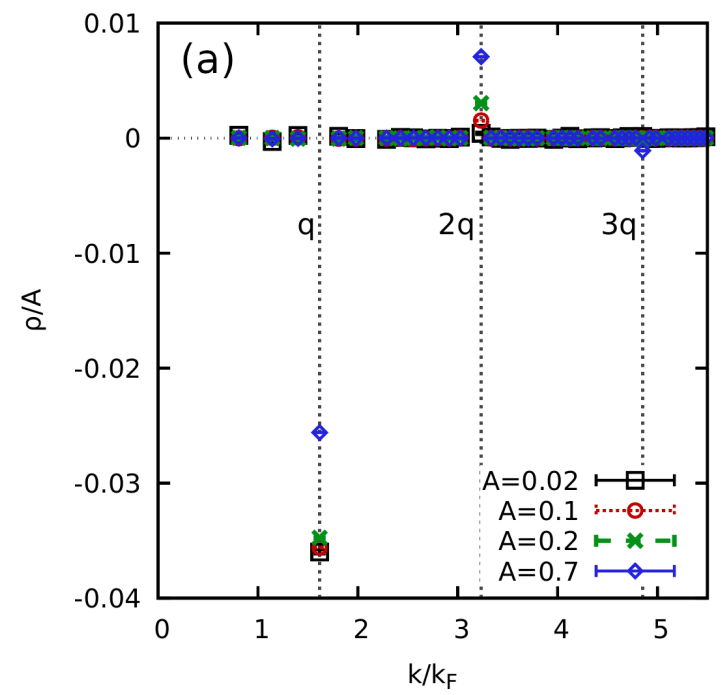

Fig. 1). At the same time, we find a significant response at the second harmonic that cannot be neglected and substantially contributes to observables like the density profile in coordinate space $n(\mathbf{r})$ that was discussed above.

A further increase of $A$ by a factor of two is depicted by the green crosses. In this case, the response at the first

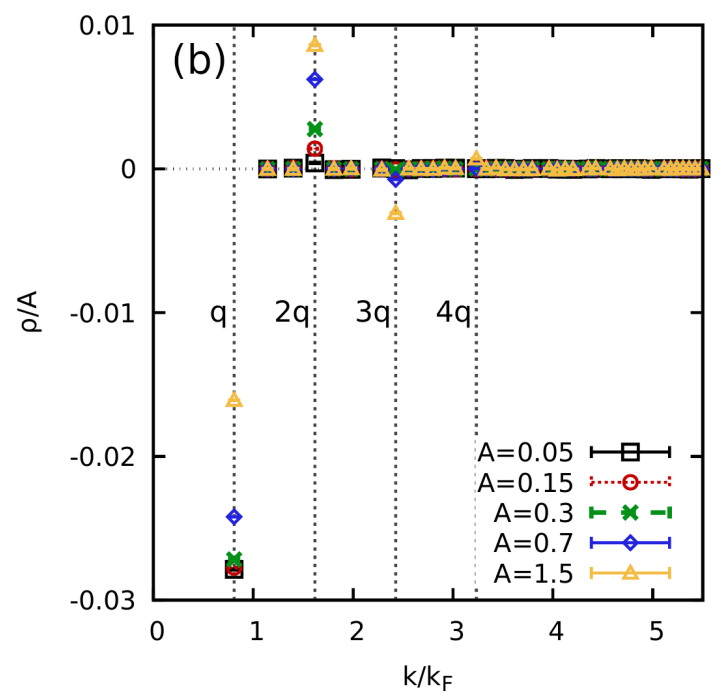

FIG. 3. Full wave-number dependence of the density response $\left\langle\hat{\rho}_{\mathbf{k}}\right\rangle_{q, A}$ of the UEG for $N=14, r_{s}=2$, and $\theta=1$ with $q \approx 1.69 q_{\mathrm{F}}$ (a) and $q \approx 0.84 q_{\mathrm{F}}$ (b) for different values of the perturbation amplitude $A$. The vertical dotted lines indicate the location of the first harmonics. 

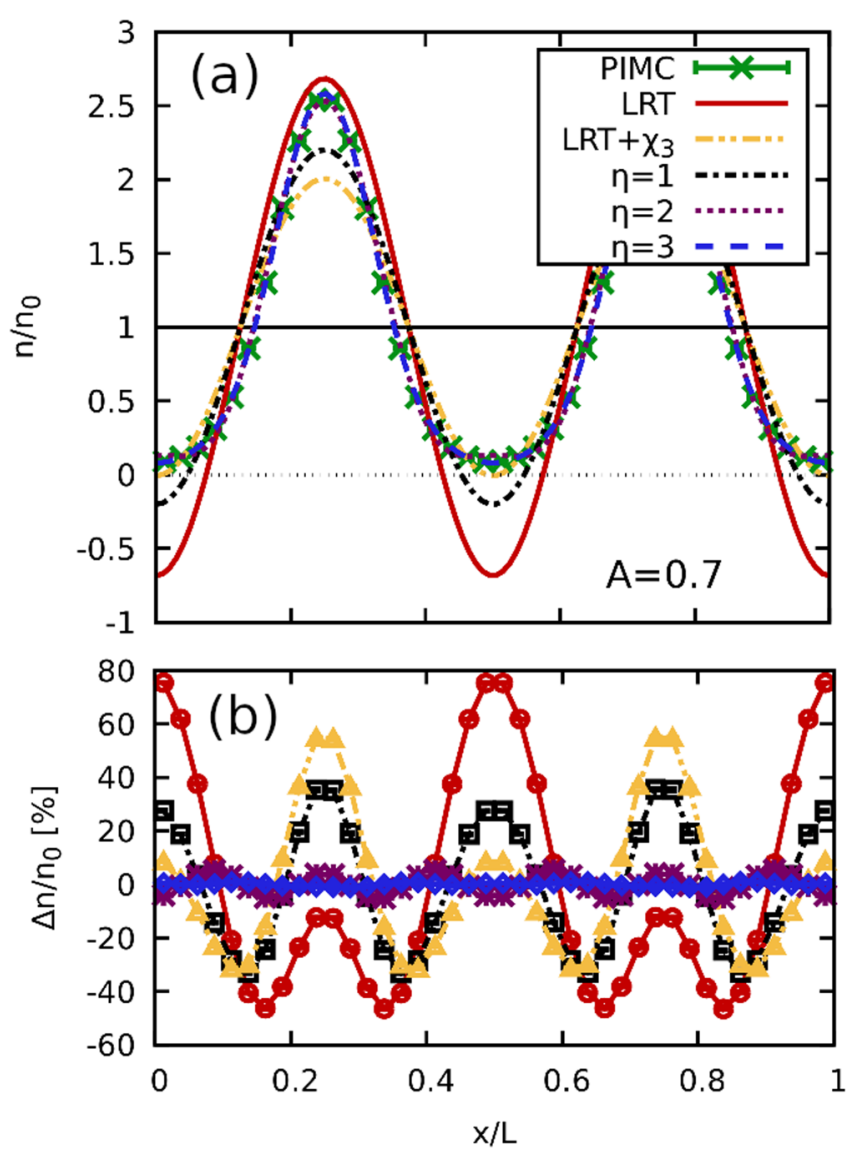

FIG. 4. Density profile of the harmonically perturbed electron gas for $N=14, r_{s}=2$, and $\theta=1$ with $q \approx 1.69 q_{\mathrm{F}}$ and $\mathbf{q}=$ $2 \pi / L(2,0,0)^{T}$ for $A=0.7$. Panel (a): Density $n(x)$ along the direction of the perturbation, with the green crosses and solid-red curves corresponding to PIMC data and LRT [cf. Eq. (7)], and the yellow dash-double-dotted, black dash-dotted, purple-dotted, and blue-dashed line corresponding to different evaluations of Eq. (20), see the main text. Panel (b) shows the corresponding deviation of different theories to the PIMC data.

harmonic clearly deviates from the prediction by LRT, and we have already seen that the cubic contribution $\chi^{(1, \text { cubic })}(q)$ is required for an adequate description. Further, we observe an even more pronounced deviation from LRT at the second harmonic, which was shown to be responsible for the bulk of the deviation between LRT and the PIMC data for $n(\mathbf{r})$ in Fig. 2. At the same time, no significant response is observed for higher harmonics $(\eta>2)$ at these conditions.

This changes for the largest depicted perturbation strength shown in Fig. 3, i.e., $A=0.7$ (blue diamonds). In this case, the signal at the first harmonic is substantially reduced compared to the other data points, and even the cubic response function $\chi^{(1, \text { cubic })}(q)$ cannot provide a reasonable description of the density response at these conditions, see Fig. 1. Moreover, we find a large signal at the second harmonic, as it is expected, and a significant contribution for the third harmonic, $k=3 q$.

The corresponding density profile in coordinate space is depicted in Fig. 4, with the green crosses again being the exact PIMC data. Evidently, the external perturbation dominates the behavior of the system under these conditions and we find an almost shell-like structure with two pronounced peaks at the positions of the minima of the harmonic potential exceeding 2.5 times the original unperturbed density $n_{0}$. These shells are separated by two deep minima where the cosinusoidal potential has its maxima.

Let us next consider the solid-red line showing the prediction by LRT, i.e., Eq. (7). Evidently, the deviation to the PIMC data is dramatic and almost attains $80 \%$ in terms of the unperturbed density $n_{0}$. In particular, LRT predicts an unphysical negative density around the minima of the density profile. Furthermore, we find that the deviation profile (bottom panel) is quickly oscillating and exhibits a complicated behavior, which means that the systematic deficiencies of LRT are both quantitative and qualitative.

The next step towards an improved description of $n(x)$ is given by the inclusion of the cubic response function $\chi^{(1, \text { cubic })}(q)$ into $\left\langle\hat{\rho}_{\mathbf{q}}\right\rangle_{q, A}$, i.e., $\left\langle\hat{\rho}_{\mathbf{q}}\right\rangle_{q, A} \approx \chi^{(1)}(q) A+$ $\chi^{(1, \text { cubic })}(q) A^{3}$. The resulting evaluation of Eq. (20) truncated at $\eta=1$ is depicted as the dash-double-dotted yellow curve and leads to a qualitative improvement of the description of the exact PIMC data, even though the deviations are still systematic and partly attain $\sim 60 \%$ of the unperturbed density $n_{0}$. This is somewhat expected as we know from Fig. 1 that even the first harmonic is not properly described by $\chi^{(1)}(q)$ and $\chi^{(1, \text { cubic })}(q)$ alone, and more terms in the expansion would be needed.

This is remedied by the dash-dotted black curve, where we again truncate Eq. (20) after $\eta=1$, but include the full PIMC expectation value for $\left\langle\hat{\rho}_{\mathbf{q}}\right\rangle_{q, A}$. On the one hand, this does not substantially improve the agreement with the exact PIMC data and there remain systematic deviation of $\sim 40 \%$. On the other hand, it makes the deviation profile shown in the bottom panel more uniform. In particular, it exhibits oscillations with twice the wave number $q$ and a nearly constant amplitude.

Naturally, these deviations are caused by the second harmonic, and including the $\eta=2$ term with the exact PIMC expectation value for $\left\langle\hat{\rho}_{2 q}\right\rangle_{q, A}$ in Eq. (20) leads to the dotted purple curve. The inclusion of the second harmonic leads to a drastic improvement, and the purple curve is in qualitative agreement with the green crosses everywhere. In particular, we note that the unphysical negative density points no longer appear. Still, the examination of the deviation profile of this curve shown in the bottom panel reveals that there remain systematic errors exceeding $5 \%$. Further, the deviation has a rapidly oscillating form with a constant amplitude, and is readily identified as the contribution of the third harmonic $k=3 q$.

In other words, it is necessary to evaluate Eq. (20) up to $\eta=3$ for such a large perturbation amplitude, and the results are shown as the dashed blue curve. Evidently, it is in excellent agreement with the PIMC data over the entire $x$ range, and the deviation plot shows that the difference between the two is fluctuating around zero within the given statistical uncertainty.

For completeness, we mention that the strong oscillations of the PIMC data in Fig. 4 are not related to the formation of a charge-density wave (CDW), which has been predicted for the UEG based on different approximations; see, e.g., Refs. [1,104-109] and references therein for more details. While the actual manifestation of a CDW in the UEG is 
still being debated in the literature, such a transition would be expected at substantially larger values of $r_{s}$. In addition we note that the density modulations observed in Fig. 4 are significantly overestimated by LRT. This, in turn, means that the nonlinear contributions actually reduce the total density response and thus tend to stabilize a uniform state even at these conditions.

Let us next consider Fig. 3(b), where we show the $k$ dependence of the density response $\left\langle\hat{\rho}_{\mathbf{k}}\right\rangle_{q, A}$ for a smaller wave number, $q \approx 0.84 q_{\mathrm{F}}$. In particular, this is the smallest wave number that is accessible within a PIMC simulation for $N=$ 14, see also Sec. II B above.

For the lowest depicted value of the perturbation amplitude ( $A=0.05$, black squares), the signal at the first harmonic $\mathbf{k}=\mathbf{q}$ cannot be distinguished from LRT [56]. In addition, we find a small yet significant signal for $\mathbf{k}=2 \mathbf{q}$, which indicates that the second harmonic is the dominant contribution to nonlinear effects in the density response of electrons in the WDM regime. This is an important finding that is discussed extensively in the context of Fig. 11 below.

For $A=0.15$ (red circles), LRT still relatively accurately describes the signal at the first harmonic, and we find a deviation to the PIMC data of less than $1 \%$. At the same time, the signal at $\mathbf{k}=2 \mathbf{q}$ is significantly increased, which further corroborates the previous observation about the respective importance of the different harmonics to the nonlinear density response.

Upon further increasing the perturbation strength to $A=$ 0.3 (green crosses), the systematic error of LRT in the description of $\left\langle\hat{\rho}_{\mathbf{q}}\right\rangle_{q, A}$ increases to $\sim 3 \%$, while the second harmonic continues to have a higher impact. Moreover, we observe a small but significant density response at the third harmonic, that was absent for $A \lesssim 0.15$.

The blue diamonds show $\left\langle\hat{\rho}_{\mathbf{k}}\right\rangle_{q, A}$ for $A=0.7$, which was the largest perturbation strength considered for $q \approx 1.69 q_{\mathrm{F}}$ shown in Fig. 3(a). For the smaller $q$ we observe a large systematic error of LRT for the first harmonic, which, however, is very accurately described by the cubic response function $\chi^{(1, \text { cubic })}(q)$. This is a direct consequence of screening effects, which make nonlinear effects less important for small wave numbers, see Ref. [56] for the first description of this finding. In the present paper, we find similar effects for the second and third harmonics as well, see below. Further, we find a large signal for $\mathbf{k}=2 \mathbf{q}$ and a smaller, but noticeable signal for $\mathbf{k}=3 \mathbf{q}$.

Finally, we consider the case of strong perturbation strength $(A=1.5)$, which is depicted by the yellow triangles. At these parameters, the density response at the first harmonic is substantially reduced compared to LRT, and the signal at $\mathbf{k}=2 \mathbf{q}$ is of the same order of magnitude. Further, the response at the third harmonic is drastically increased compared to $A=0.7$, and we even find a significant response for the fourth harmonic $\mathbf{k}=4 \mathbf{q}$.

Let us proceed with a more rigorous quantification of the nonlinear electronic density response by obtaining the respective generalized response functions defined in Eqs. (36)-(38). For the case of the cubic response function of the first harmonic $\chi^{(1, \text { cubic })}(q)$, this is demonstrated in Fig. 5 for three different wave numbers. More specifically, we show PIMC results for the dependence of $\left\langle\hat{\rho}_{\mathbf{q}}\right\rangle_{q, A}$ on the perturbation

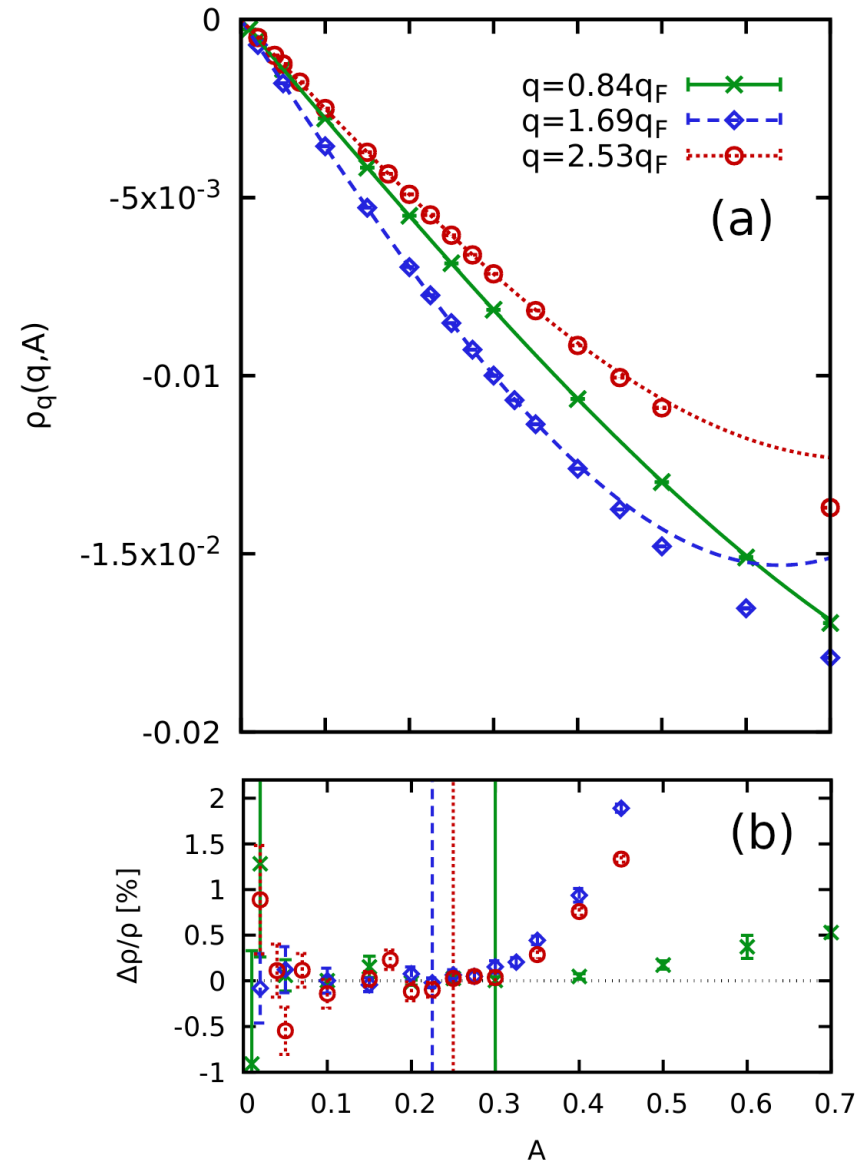

FIG. 5. Determination of the cubic response function $\chi^{(1, \text { cubic })}(q)$. Panel (a): Density response of the UEG for $N=14$, $r_{s}=2$, and $\theta=1$ for $q \approx 0.84 q_{\mathrm{F}}$ (green crosses) $q \approx 1.69 q_{\mathrm{F}}$ (blue diamonds), and $q \approx 2.53 q_{\mathrm{F}}$ (red circles). The corresponding curves of the same color have been obtained by fitting Eq. (36) to the PIMC data. Panel (b): Relative deviation of the fits to the PIMC data. The vertical lines show the respective maximum value of $A$ that was included in the fits.

strength for $q \approx 0.84 q_{\mathrm{F}}$ (green crosses), $q \approx 1.69 q_{\mathrm{F}}$ (blue diamonds), and $q \approx 2.53 q_{\mathrm{F}}$ (red circles), and the corresponding curves show the respective fits according to Eq. (36). Panel (b) shows the deviation between fits and PIMC data and the vertical lines indicate the respective maximum values of $A$ up to which data have been included in the fitting procedure.

Let us briefly touch upon the $A$ dependence of the Monte Carlo error bars in the deviation plot. For weak perturbations $A$, the absolute values of the density response $\left\langle\hat{\rho}_{\mathbf{q}}\right\rangle_{q, A}$ are small, which means that the denominator of the relative deviations shown in the bottom of Fig. 5 is small, too. At the same time, the absolute values of the Monte Carlo error bars are nearly independent of $A$, which means that the relative accuracy of our PIMC data for Eq. (3) decreases for weak perturbations, and, eventually, the density response cannot be resolved within the given statistical uncertainty.

Regarding the $A$ dependence of $\left\langle\hat{\rho}_{\mathbf{q}}\right\rangle_{q, A}$ itself, we find that the blue diamonds exhibit the largest density response at the first harmonic, whereas the signal is weakest for the largest wave number, $q=2.53 q_{\mathrm{F}}$. While not being trivial, this is 


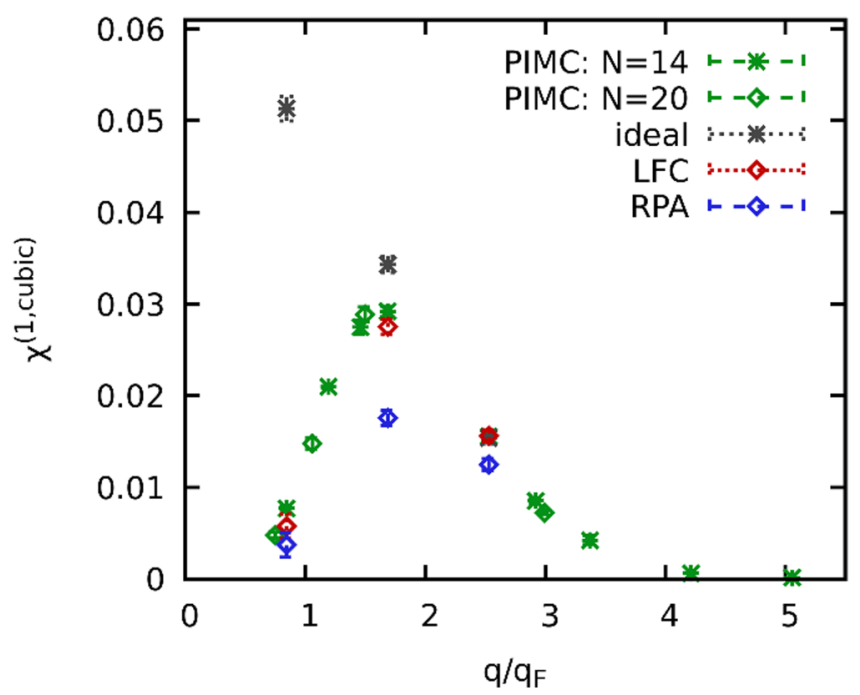

FIG. 6. Wave-number dependence of the cubic response function of the first harmonic $\chi^{(1, \text { cubic })}(q)$ for the warm dense electron gas at $r_{s}=2$ and $\theta=1$.

somewhat expected as the LRT function $\chi^{(1)}(q)$, too, exhibits a maximum response around $q \sim 1.5 q_{\mathrm{F}}$, cf. Fig. 17 below. Interestingly, Eq. (36) remains accurate for larger perturbation amplitudes $A$ for the green crosses compared to the other two cases. This can be explained by the comparatively smaller value of $\chi^{(1, \text { cubic })}$ in this case, see Fig. 6.

Repeating the fitting procedure demonstrated in Fig. 5 for different values of the perturbation wave numbers gives us access to the full $q$ dependence of $\chi^{(1, \text { cubic })}(q)$, and the results are shown in Fig. 6. More specifically, the green data points have been obtained from our PIMC simulations and the stars and diamonds show results for $N=14$ and $N=20$ particles. While being available at different discrete $q$ points $[41,110]$, we note that no dependence on the system size can be resolved within the given confidence intervals [56]. This is expected as previous studies of related properties such as the static structure factor $S(q)$ [41,111], dynamic structure factor $S(q, \omega)[78]$ and the linear response function $\chi^{(1)}(q)$ [58], too, have found finite-size effects to be small in this regime. For completeness, we mention that this changes towards lower temperature, where momentum shell effects [112] induce a more pronounced dependence on $N$. The grey stars, too, have been obtained from PIMC data, but for $N=14$ ideal (noninteracting) fermions. In this case, there are no screening effects and $\chi_{0}^{(1, \text { cubic })}(q)$ attains a finite value in the limit of $q \rightarrow 0$.

Unfortunately, no simple recursion relation is available for the ideal function $\chi_{0}^{(1, \text { cubic })}(q)$ [see Sec. II above], so that we cannot evaluate the RPA and LFC expressions for the cubic response at the first harmonic given in Eqs. (31) and (35) for all wave numbers. Yet, we can still use the three PIMC data points, and the results are depicted by the blue (RPA) and red (LFC) data points in Fig. 6. Evidently, the RPA curve seems to qualitatively reproduce the correct behavior, but severely underestimates the true magnitude of the cubic response around its maximum. In contrast, the LFC curve nicely agrees with the PIMC data points for the interacting systems for all three wave numbers.
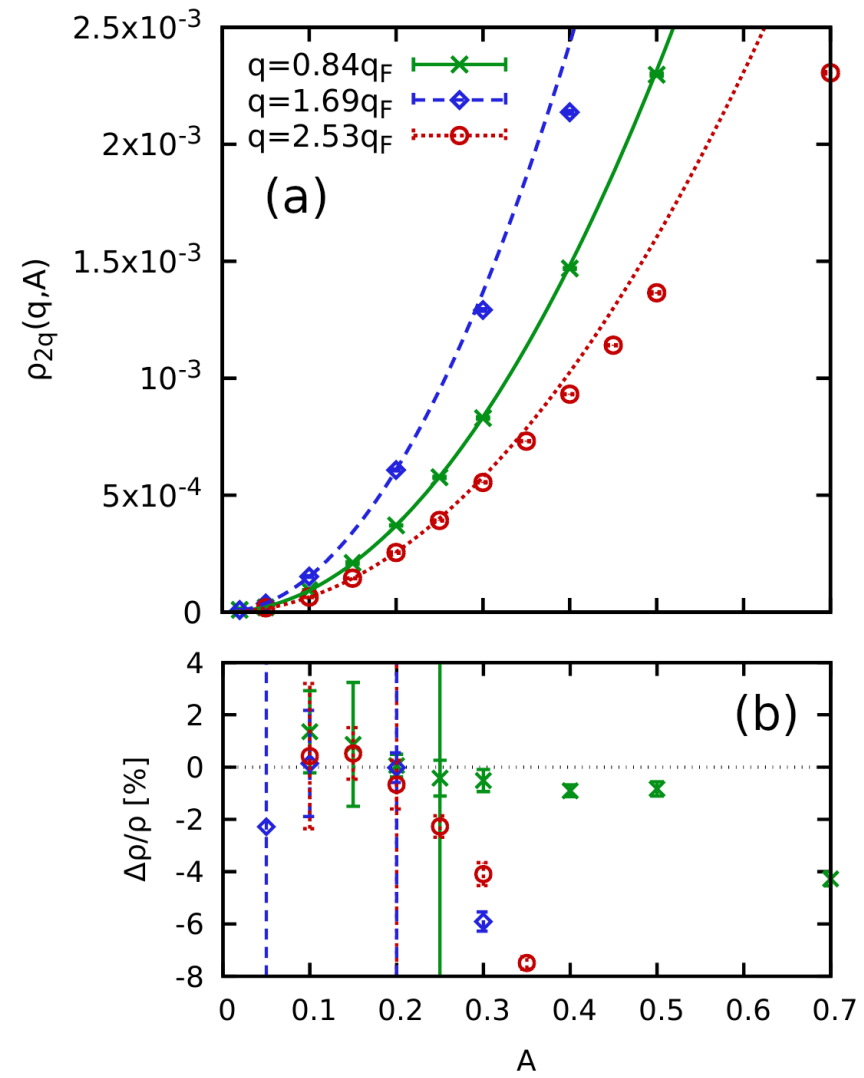

FIG. 7. Determination of the quadratic response function $\chi^{(2)}(q)$. Panel (a): Density response of the second harmonic $\left\langle\hat{\rho}_{2 \mathbf{q}}\right\rangle_{q, A}$ of the $\mathrm{UEG}$ for $N=14, r_{s}=2$, and $\theta=1$ for $q \approx 0.84 q_{\mathrm{F}}$ (green crosses) $q \approx 1.69 q_{\mathrm{F}}$ (blue diamonds), and $q \approx 2.53 q_{\mathrm{F}}$ (red circles). The corresponding curves of the same color have been obtained by fitting Eq. (37) to the PIMC data. Panel (b): Relative deviation of the fits to the PIMC data. The vertical lines show the respective maximum value of $A$ that was included in the fits.

Let us next repeat this analysis for the quadratic response function of the second harmonic $\chi^{(2)}(q)$, that has been shown to constitute the dominant nonlinear effect above. To this end, we show the $A$ dependence of the density response of the second harmonic $\left\langle\hat{\rho}_{2 \mathbf{q}}\right\rangle_{q, A}$ in Fig. 7 for the same wave numbers as in Fig. 5.

Firstly, we observe the same absolute ordering of the three data sets with $q$ as for the first harmonic, with $q=1.69 q_{\mathrm{F}}$ $\left(q=0.84 q_{\mathrm{F}}\right)$ exhibiting the largest (weakest) signal for the density response. Further, we see that the parabolic expansion given in Eq. (37) is in excellent agreement with the PIMC data points for small $q$, which gives us direct access to the corresponding quadratic density response function.

The results for the full wave-number dependence of $\chi^{(2)}(q)$ are shown in Fig. 8 with the same key as $\chi^{(1, \text { cubic })}(q)$ shown in Fig. 6 above. There is excellent agreement between the exact quadratic response function of the ideal Fermi gas [yellow curve, cf. Eq. (21)] and our PIMC data for $N=14$ noninteracting fermions (grey stars) over the entire depicted $q$ range. In addition, we find that the quadratic response function of the UEG $\chi^{(2)}(q)$ exhibits a qualitatively similar behavior to $\chi^{(1, \text { cubic })}(q)$ shown above. More specifically, $\chi^{(2)}(q)$ vanishes for small wave numbers due to the perfect screening in the 


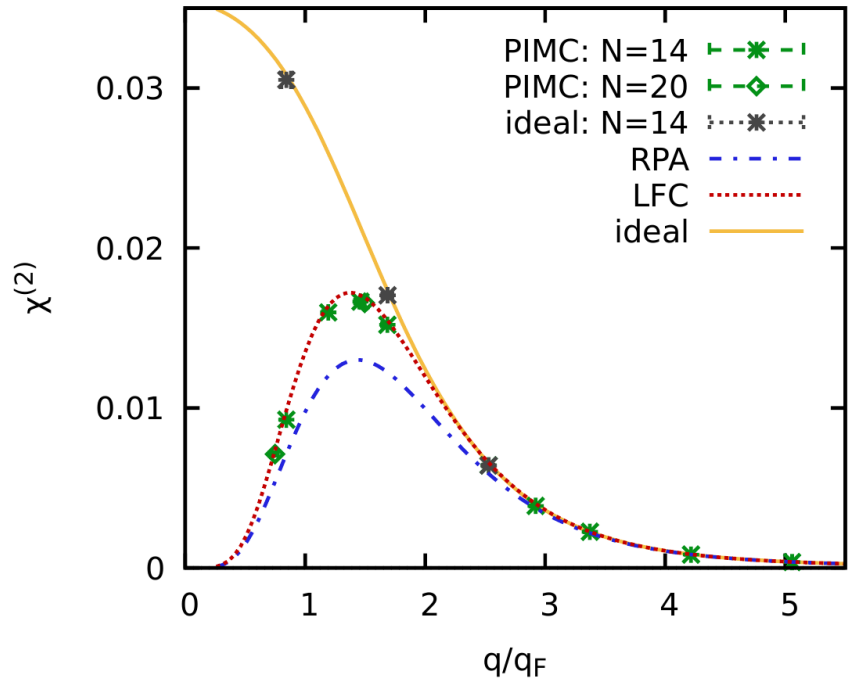

FIG. 8. Wave-number dependence of the quadratic response function of the second harmonic $\chi^{(2)}(q)$ for the warm dense electron gas at $r_{s}=2$ and $\theta=1$. The green stars (green diamonds) show PIMC results obtained from Eq. (37) for $N=14(N=20)$ electrons, and the grey stars to PIMC data for ideal (noninteracting) fermions. The dashed blue and dotted-red lines show theoretical results within RPA [Eq. (24)] and using the static LFC from Ref. [58] [Eq. (33)], and the solid-yellow line shows the ideal response function $\chi_{0}^{(2)}(q)$ [Eq. (21)].

UEG, and, too, vanishes in the limit of large $q$, albeit slower than for $q \rightarrow 0$.

The dashed-blue RPA curve [Eq. (24)] is in qualitative agreement with our PIMC data for the UEG (green stars and diamonds) and correctly reproduces both of these limits. Yet, there appear systematic deviations exceeding $20 \%$ for intermediate wave numbers $q \sim 1.5 q_{\mathrm{F}}$ (i.e., in the vicinity of the maximum), which resembles the known deficiencies of RPA within LRT, see, e.g., Refs [36,58,113].

Finally, the dotted red curve has been obtained by including the neural-net representation of the static LFC $G(q)$ given by Dornheim et al. [58] [Eq. (33)] and is in excellent agreement with the PIMC data for all $q$.

The last generalized response function to be considered in this work is the cubic response function of the third harmonic $\chi^{(3)}(q)$, that can be obtained by fitting Eq. (38) to PIMC data for $\left\langle\hat{\rho}_{3 \mathbf{q}}\right\rangle_{q, A}$. This is shown in Fig. 9, where the $A$ dependence of the density response at $\mathbf{k}=3 \mathbf{q}$ is shown for the same three wave numbers as for the other response functions investigated above. In this case, we observe a comparatively large increase of $\left\langle\hat{\rho}_{\mathbf{3 q}}\right\rangle_{q, A}$ with $A$ at $q=1.69 q_{\mathrm{F}}$ that is approximately four times as large as for the other two wave numbers, which are similar in magnitude.

Moreover, we note that the absolute value of the density response at $\mathbf{k}=3 \mathbf{q}$ is an order of magnitude smaller than for $\mathbf{k}=2 \mathbf{q}$ for the same values of the perturbation amplitude $A$. This, in turn, means that the relative error bars in $\left\langle\hat{\rho}_{\mathbf{3 q}}\right\rangle_{q, A}$ are larger than for the other harmonics shown above, which makes the determination of $\chi^{(3)}(q)$ more challenging.

The corresponding wave-number dependence of this function is shown in Fig. 10. Let us first focus on the noninteract-

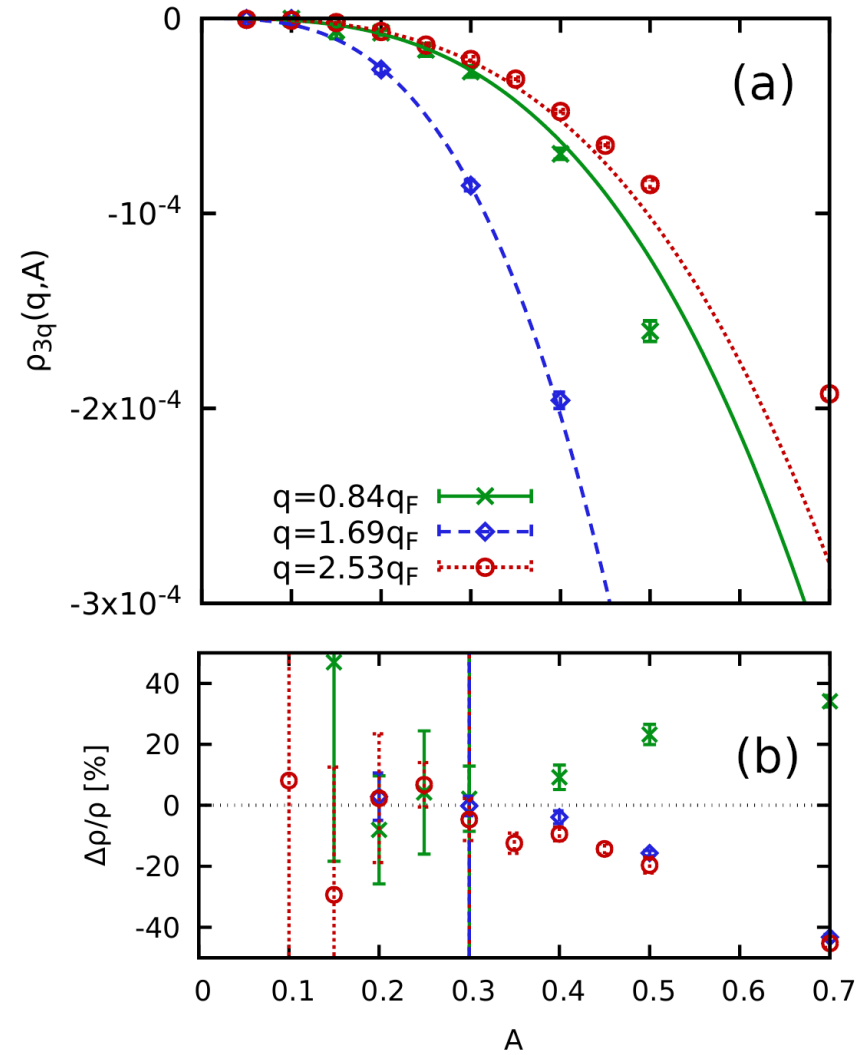

FIG. 9. Determination of the cubic response function $\chi^{(3)}(q)$. Panel (a): Density response of the third harmonic $\left\langle\hat{\rho}_{3 \mathbf{q}}\right\rangle_{q, A}$ of the UEG for $N=14, r_{s}=2$, and $\theta=1$ for $q \approx 0.84 q_{\mathrm{F}}$ (green crosses) $q \approx 1.69 q_{\mathrm{F}}$ (blue diamonds), and $q \approx 2.53 q_{\mathrm{F}}$ (red circles). The corresponding curves of the same color have been obtained by fitting Eq. (38) to the PIMC data. Panel (b): Relative deviation of the fits to the PIMC data. The vertical lines show the respective maximum value of $A$ that was included in the fits.

ing case, with the grey stars depicting PIMC results for $N=$ 14 ideal fermions and the solid yellow curve the exact ideal response function $\chi_{0}^{(3)}(q)$ that we obtain from Eq. (22). Remarkably, even without the electronic Coulomb repulsion and the associated screening effects, the density response function of the third harmonic exhibits a complicated, nonmonotonous behavior, with a maximum in magnitude around the Fermi wave number. This is confirmed by the corresponding ideal PIMC data point at $q=0.84 q_{\mathrm{F}}$, which nicely agrees with the exact result.

For the interacting UEG, this interesting behavior at small $q$ is masked by screening effects, and the generalized response function exhibits similar trends as $\chi^{(1, \text { cubic })}(q)$ and $\chi^{(2)}(q)$, that is, it vanishes both in the limits of small and large $q$. As seen before, the RPA of the cubic response at the third harmonic underestimates the response, but the inclusion of LFCs gives a nice agreement between QMC simulations and theory.

Let us conclude this section with a systematic investigation of the respective contribution of the different generalized response functions to the total nonlinear density response. In Fig. 11, we show the relative contribution of $\chi^{(2)}(q)$ (blue), $\chi^{(1, \text { cubic) }}(q)$ (green), and $\chi^{(3)}(q)$ (black) compared to the 


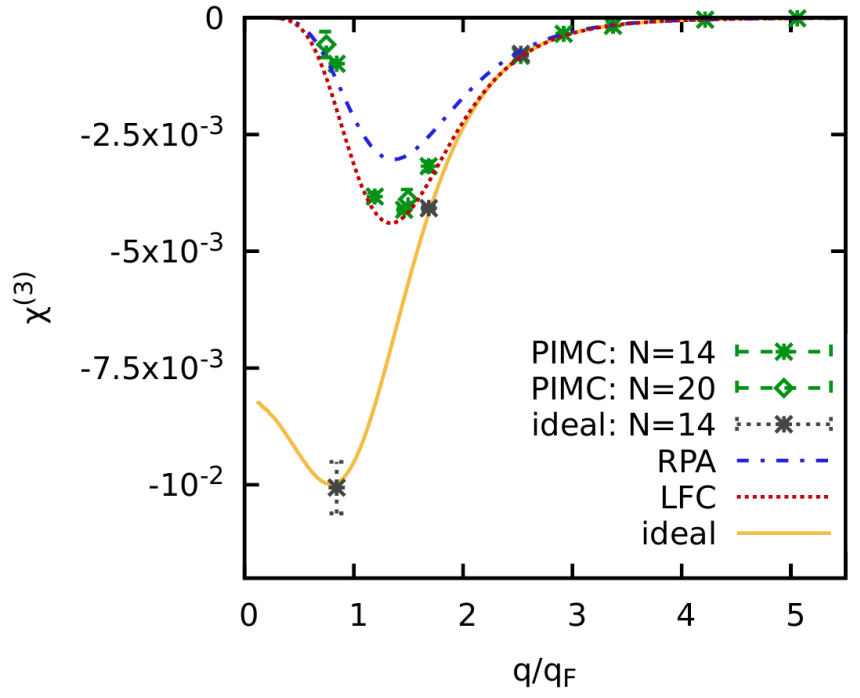

FIG. 10. Wave-number dependence of the cubic response function of the third harmonic $\chi^{(3)}(q)$ for the warm dense electron gas at $r_{s}=2$ and $\theta=1$. The green stars (green diamonds) show PIMC results obtained from Eq. (38) for $N=14(N=20)$ electrons, and the grey stars to PIMC data for ideal (noninteracting) fermions. The dashed-blue and dotted-red lines show theoretical results within RPA [Eq. (30)] and using the static LFC from Ref. [58] [Eq. (34)], and the solid yellow line shows the ideal response function $\chi_{0}^{(3)}(q)$ [Eq. (22)].

density response at the first harmonic predicted by LRT, Eq. (4). More specifically, the data points have been obtained from fits to PIMC data, and the dotted (dashed) lines are theoretical results including an LFC (RPA only).

Panel (a) of Fig. 11 corresponds to a weak perturbation, $A=0.02$. At these conditions, LRT is relatively accurate (see, e.g., the left panel of Fig. 2) and the response due to the cubic response functions for both the first and third harmonic is smaller than $0.1 \%$ compared to LRT. By far the largest nonlinear effect can be observed at the second harmonic with a maximum signal of almost $1 \%$ in terms of $\rho_{\mathrm{LRT}}$. In particular, the response for $\mathbf{k}=2 \mathbf{q}$ is more than an order of magnitude larger than the other two depicted curves.

Figure 11(b) corresponds to a stronger perturbation, $A=$ 0.2 . This is a particularly interesting example, as the density response for the first three harmonics is fully described by the different response functions at these conditions. In contrast, additional terms of the respective expansions in powers of $A$ would be needed for an accurate description of $\left\langle\hat{\rho}_{\mathbf{k}}\right\rangle_{q, A}$ at much larger values of $A$.

Let us first consider the density response of the second harmonic, which again constitutes the largest nonlinear effect with a maximum contribution of almost $10 \%$ around $q \sim$ $1.5 q_{\mathrm{F}}$ compared to Eq. (4). Further, we note that the relative impact of $\left\langle\hat{\rho}_{2 \mathbf{q}}\right\rangle_{q, A}$ only diminishes in the small wave-number limit for $q \lesssim 0.2 q_{\mathrm{F}}$, and disappears even more slowly for large values of $q$, with $\left\langle\hat{\rho}_{2 \mathbf{q}}\right\rangle_{q, A} / \rho_{\mathrm{LRT}}$ exceeding $1 \%$ even at $q=5 q_{\mathrm{F}}$

In addition, the cubic contribution to the density response at the first harmonic $\left\langle\Delta \hat{\rho}_{\mathbf{q}}\right\rangle_{q, A}=\chi^{(1, \text { cubic })}(q) A^{3}$ is substantially more important compared to $A=0.02$, and we find a maximum impact of approximately $4 \%$ around $q=1.5 q_{\mathrm{F}}$.
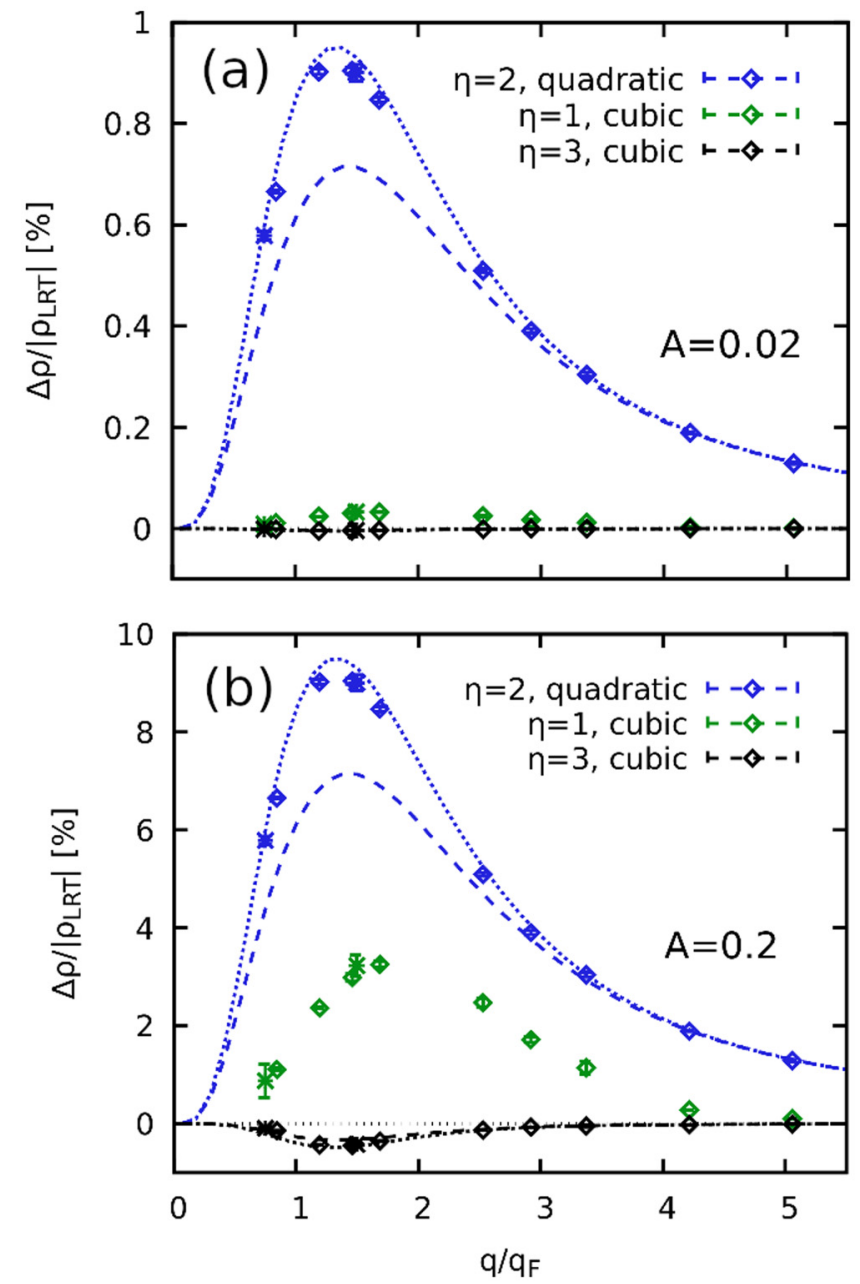

FIG. 11. Relative impact of different nonlinear contributions to the density response $\left\langle\hat{\rho}_{\mathbf{q}}\right\rangle$ compared to the prediction for $\left\langle\hat{\rho}_{\mathbf{q}}\right\rangle_{q, A}$ from LRT for $A=0.02$ (a) and $A=0.2$ (b). Shown are $\left\langle\hat{\rho}_{2 \mathbf{q}}\right\rangle_{q, A}=$ $\chi^{(2)}(q) A^{2}$ (blue), $\left\langle\hat{\rho}_{3 \mathbf{q}}\right\rangle_{q, A}=\chi^{(3)}(q) A^{3}$ (black), and $\left\langle\Delta \hat{\rho}_{\mathbf{q}}\right\rangle_{q, A}=$ $\chi^{(1, \text { cubic })}(q) A^{3}$ (green). The dashed and dotted lines have been obtained within RPA and using a LFC, respectively.

Indeed, this is the same order of magnitude as the signal of the second harmonic, which means that both $\chi^{(2)}(q)$ and $\chi^{(1, \text { cubic })}(q)$ have to be taken into account for an accurate description of the system, cf. the right column of Fig. 2 above showing the radial density $n(\mathbf{r})$ for this case. At the same time, we find that the impact of the cubic contribution to the first harmonic rapidly vanishes in both the limits of large and small wave numbers and is only relevant for intermediate $q$.

Finally, the black points and curves correspond to the contribution of the third harmonic, which is an order of magnitude smaller than the green curve. Therefore, it appears to be mostly negligible for practical purposes.

\section{B. Temperature dependence}

Let us next turn our attention to the dependence of nonlinear effects in the static density response on the temperature $\theta$. In Fig. 12(a), we show the density profile in coordinate space $n(\mathbf{r})$ along the direction of the perturbation, for $N=14$ electrons at $r_{s}=2$ for $q \approx 0.84 q_{\mathrm{F}}$ and a relatively small value 



FIG. 12. Density profile of the harmonically perturbed electron gas for $N=14$ and $r_{s}=2$, with $q \approx 0.84 q_{\mathrm{F}}$ and $\mathbf{q}=2 \pi / L(1,0,0)^{T}$ for $A=0.1$. Panel (a): PIMC results for the density $n(x)$ along the direction of the perturbation for $\theta=1$ (blue diamonds), $\theta=2$ (red circles), and $\theta=4$ (green crosses). The solid curves correspond to LRT [cf. Eq. (7)]. Panel (b): Relative deviation of LRT to the PIMC data.

of the perturbation amplitude $A=0.1$. The blue diamonds have been obtained for $\theta=1$ and exhibit a deviation from the unperturbed density $n_{0}$ of almost $20 \%$ at the maxima and minima. The solid-blue curve shows the prediction from LRT [cf. Eq. (7)] and qualitatively captures the correct behavior. This can again be seen particularly well in panel (b), where we show the relative deviation between the PIMC data and LRT. Evidently, LRT results in an oscillating deviation profile that is mainly due to the density response at the second harmonic $\left\langle\hat{\rho}_{2 \mathbf{q}}\right\rangle_{q, A}$ (see the previous section III A), with a maximum deviation below $0.8 \%$.

Let us next focus on the red circles corresponding to twice the temperature, $\theta=2$. Firstly, we note that the overall density response is reduced compared to $\theta=1$, as it is expected, see also the corresponding discussion in Refs. [56,113]. In particular, an increasing temperature leads to an increased internal energy scale of the system, which, in turn, means that the relative impact of an external potential is reduced if the perturbation amplitude $A$ is kept constant. The green crosses correspond to a further increase of the temperature by a factor of two, and the total density response is again reduced compared to the other data sets.
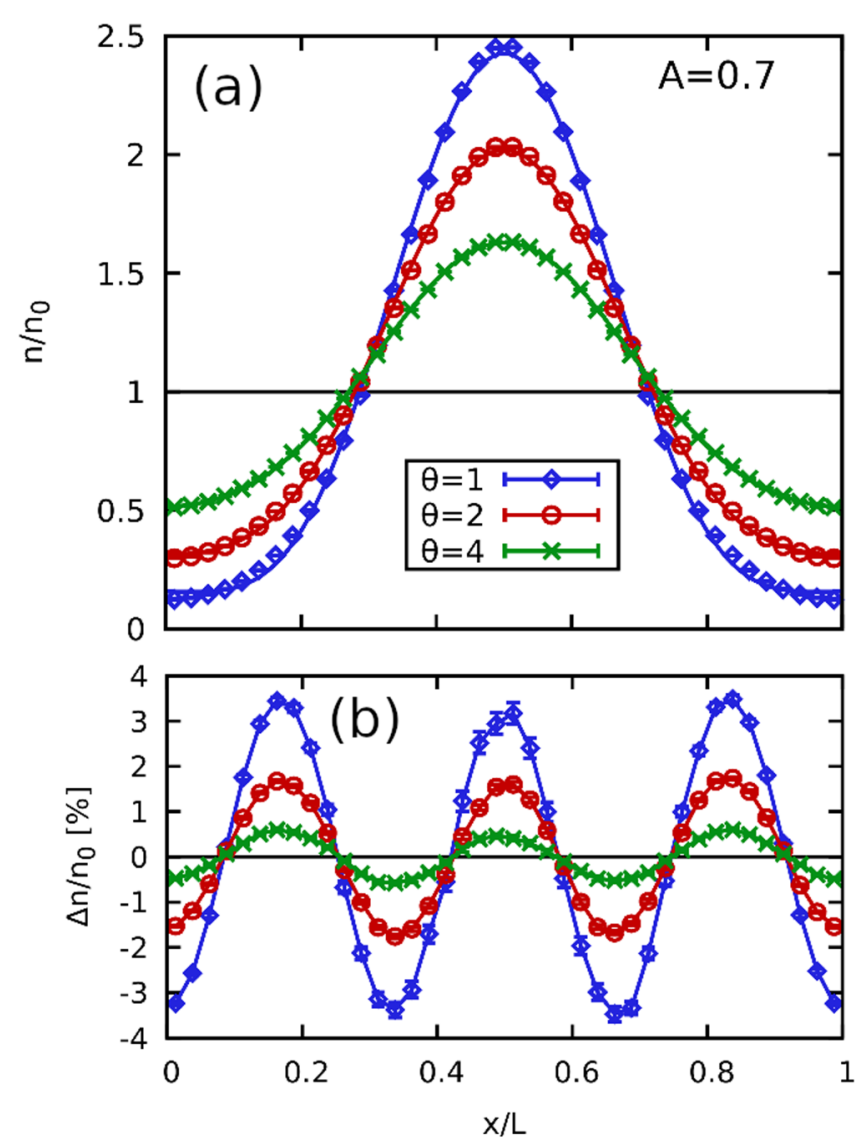

FIG. 13. Density profile of the harmonically perturbed electron gas for $N=14$ and $r_{s}=2$, with $q \approx 0.84 q_{\mathrm{F}}$ and $\mathbf{q}=2 \pi / L(1,0,0)^{T}$ for $A=0.7$. Panel (a): PIMC results for the density $n(x)$ along the direction of the perturbation for $\theta=1$ (blue diamonds), $\theta=2$ (red circles), and $\theta=4$ (green crosses). The solid curves correspond to the evaluation of Eq. (20) truncated after $\eta=2$. Panel (b): Relative deviation of solid curves from the PIMC data.

In addition, a reduced density response means that nonlinear effects become less important and we find maximum deviations between LRT and the PIMC data of $0.4 \%(0.2 \%)$ for $\theta=2(\theta=4)$. At the same time, we note that the nonlinear response is significant for all three depicted temperatures and is mainly given by the signal at the second harmonic, $\mathbf{k}=2 \mathbf{q}$.

Let us next consider Fig. 13, which shows the same information as Fig. 12, but for a significantly increased perturbation strength $A=0.7$. In this case, the density response is strongly nonlinear for all three depicted temperatures, although the density profile $n(\mathbf{r})$ is substantially different for all three cases. For $\theta=1$, the external perturbation constitutes the dominant effect and fully shapes the physical behavior of the system. Most particles are, on average, located around the center of the simulation cell where the cosinusoidal potential has its minimum, whereas the density is reduced by roughly $90 \%$ for small and large $x$. The solid-blue curve has been computed by truncating Eq. (20) after the second harmonic $(\eta=2)$ using the exact PIMC expectation values for the coefficients $\left\langle\hat{\rho}_{\mathbf{q}}\right\rangle_{q, A}$ and $\left\langle\hat{\rho}_{2 \mathbf{q}}\right\rangle_{q, A}$. In this case, we find that the agreement to the PIMC data is only very qualitative, and 

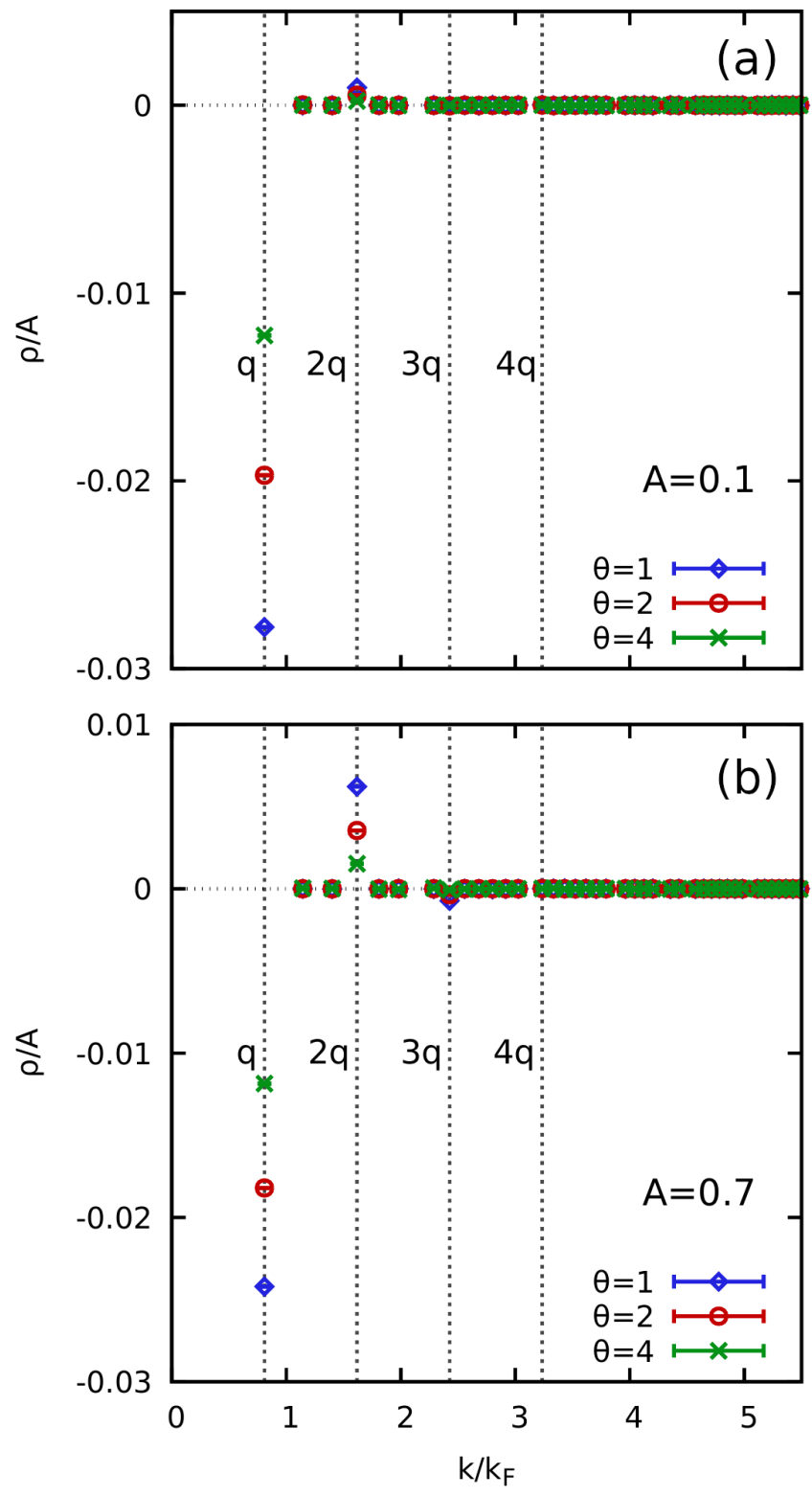

FIG. 14. Full wave-number dependence of the density response $\left\langle\hat{\rho}_{\mathbf{k}}\right\rangle_{q, A}$ of the UEG for $N=14, r_{s}=2$, and $\theta=1$ with $q \approx 0.84 q_{\mathrm{F}}$ and $A=0.1$ (a) and $A=0.7$ (b). Shown are PIMC results for Eq. (3) for $\theta=1$ (blue diamonds), $\theta=2$ (red circles), and $\theta=4$ (green crosses). The vertical dotted lines indicate the location of the first four harmonics.

there appear rapidly oscillating deviations [panel (b)] with an amplitude exceeding 3\%. Naturally, these deviations constitute the signal of the third harmonic, $\mathbf{k}=3 \mathbf{q}$, see also the discussion of Fig. 14 below.

For $\theta=2$, the density at the maxima of the external perturbation is substantially larger compared to the lower temperature, and the impact of the third harmonic on the density profile $n(\mathbf{r})$ does not exceed $2 \%$. Finally at the highest temperature, we find oscillations in the total density $n$ of approximately $50 \%$, which is substantially smaller compared to the other cases. Consequently, the first two harmonics already provide a rather accurate description, and the systematic error is smaller than $1 \%$ everywhere.

A more systematic investigation of the respective strength of the density response at different harmonics is shown in Fig. 14, where we show the full $\mathbf{k}$ dependence of $\left\langle\hat{\rho}_{\mathbf{k}}\right\rangle_{q, A}$ for the two examples investigated in Figs. 12 and 13. Panel (a) corresponds to the weaker perturbation, $A=0.1$. We note that we again only observe a nonzero signal for the integer harmonics of $\mathbf{q}$, as it is expected. For the first harmonic $(\mathbf{k}=\mathbf{q})$, we see the expected order of the signals for the three depicted temperatures, with the response at $\theta=4$ being less than half the response at $\theta=1$.

For the second harmonic $(\mathbf{k}=2 \mathbf{q})$, the relative strength of the signals for different temperatures is even more different, with the response for $\theta=4(\theta=2)$ being approximately $25 \%$ $(50 \%)$ of the response for $\theta=1$.

Finally, we mention that the signal at the third harmonic $(\mathbf{k}=3 \mathbf{q})$ vanishes within the given level of accuracy for all $\theta$.

Figure 14(b) shows the same results for $A=0.7$ and we find similar trends. In fact, the ratio of density response for $\theta=2$ and $\theta=4$ for both the first and second harmonic compared to the respective response at $\theta=1$ is remarkably unchanged, although the total response at $\mathbf{k}=2 \mathbf{q}$ has substantially increased. Moreover, we find a nonzero signal at $\mathbf{k}=3 \mathbf{q}$ for all three temperatures, whereas the response at the fourth harmonic vanishes even for $\theta=1$.

The next step towards a more complete description of the temperature dependence of the nonlinear density response is given by the determination of the different generalized response functions. To this end, we show the dependence of the density response at the second harmonic $\left\langle\hat{\rho}_{2 \mathbf{q}}\right\rangle_{q, A}$ on the perturbation amplitude $A$ in Fig. 15 for the same conditions as in the previous figures in this section. The points depict PIMC data for the three different temperatures, and the curves parabolic fits to these data according to Eq. (37). Panel (b) shows the relative deviation between PIMC and the fits, and the vertical bars show the respective maximum value of $A$ up to which points have been included into the fitting procedure. Evidently, Eq. (37) is capable to accurately reproduce the PIMC data for $A \lesssim 0.4$ in all three cases. Going back to panel (a), we see that both the PIMC results and the parabolic fits are systematically ordered with $\theta$, as it is expected.

Repeating this analysis for different values of the perturbation wave number $q$ gives us access to the full wave-number dependence of the quadratic response function of the second harmonic $\chi^{(2)}(q)$, and the results are shown in Fig. 16 for different relevant temperatures. The symbols have been obtained on the basis of fitting Eq. (37) to PIMC data, and the solid [dashed] curves correspond to the theory with a LFC [within RPA], cf. Eq. (33) [Eq. (24)].

First and foremost, we note that $\chi^{(2)}$ exhibits a substantial dependence on $\theta$, and the maximum value of this function at $\theta=0.01$ (black) is larger by an order of magnitude compared to $\theta=4$ (green). Moreover, the shape of the curves strongly depends on $\theta$ as well: while the curves for all temperatures are relatively similar both in the limit of small and large wave numbers $q$, there emerges a sharp peak around $q \sim 1.3 q_{\mathrm{F}}$ with decreasing $\theta$. 


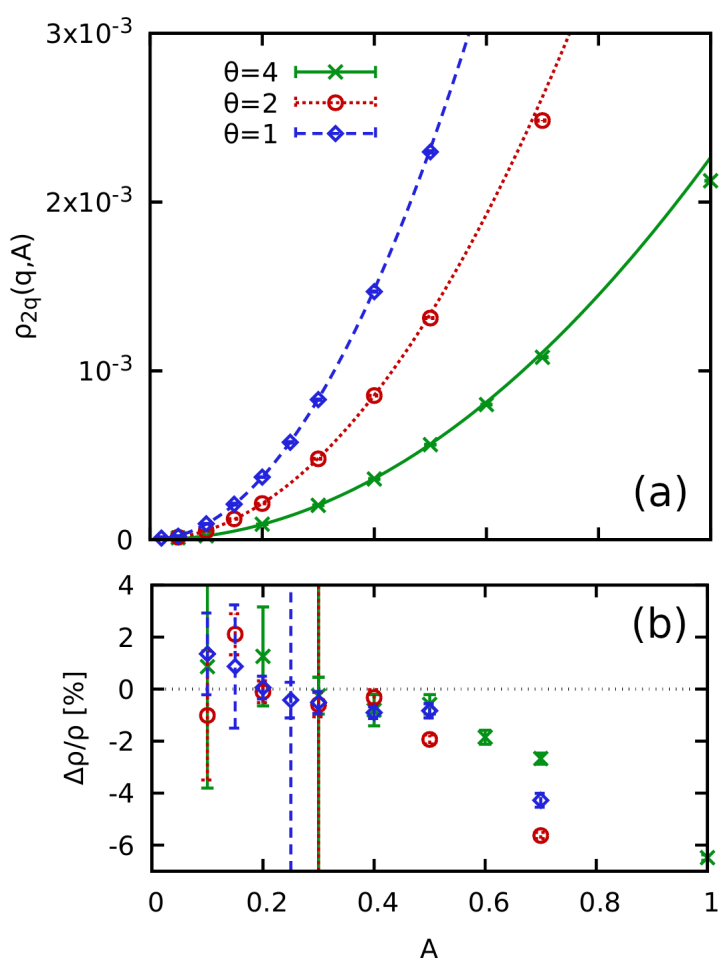

FIG. 15. Determination of the quadratic response function $\chi^{(2)}(q)$. Panel (a): Density response of the second harmonic $\left\langle\rho_{2 \mathbf{q}}\right\rangle_{q, A}$ of the UEG for $N=14, r_{s}=2$, and $q \approx 0.84 q_{\mathrm{F}}$ for $\theta=1$ (blue diamonds) $\theta=2$ (red circles), and $\theta=4$ (green crosses). The corresponding curves of the same color have been obtained by fitting Eq. (37) to the PIMC data. Panel (b): Relative deviation of the fits to the PIMC data. The vertical lines show the respective maximum value of $A$ that was included in the fits.

Let us next analyze the level of accuracy of both RPA and LFC, which, too, strongly depends on $\theta$. For $\theta=4$, the system is only weakly coupled and both RPA and LFC are in very good agreement to the PIMC data (green crosses) over the entire depicted wave-number range. Upon decreasing the temperature to $\theta=2$, RPA systematically underestimates the quadratic response function in the vicinity of its maximum, whereas the LFC still nicely reproduces the PIMC results (red circles) everywhere. For $\theta=1$, the deviation between RPA and LFC becomes substantial and partly exceeds $20 \%$, whereas the LFC gives both a qualitatively and quantitatively sufficient description. At the same time, we like to reiterate the point that Eq. (33) does only include screening effects to a linear order, and, thus does not give an exact description even with the exact static limit of the LFC $G(q)$ taken from Ref. [58]. Indeed, the systematic inaccuracy of the approximate description of screening effects in Eq. (33) increases with the effective coupling strength of the system. With the density parameter $r_{s}$ being constant, the effective coupling increases towards small temperature and small deviations between the PIMC data points and the theoretical prediction become noticeable for $\theta \lesssim 1$.

Finally, the black curves show results for $\theta=0.01$, where the system is in the ground state. In this case, the systematic deviation between LFC and RPA is even larger compared

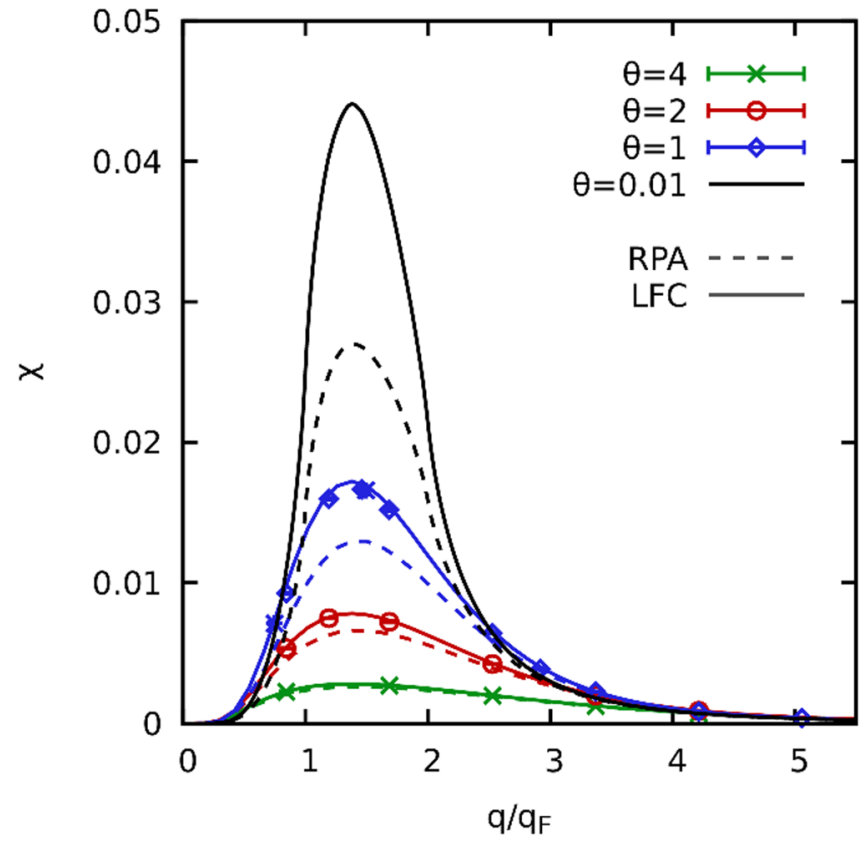

FIG. 16. Wave-number dependence of the quadratic response function of the second harmonic $\chi^{(2)}(q)$ for the warm dense electron gas at $r_{s}=2$ and $\theta=1$ (blue diamonds), $\theta=2$ (red circles), and $\theta=4$ (green crosses). The dashed and solid lines show theoretical results within RPA and using the LFC from Ref. [58], cf. Eqs. (24) and (32).

to $\theta=1$, as the effective coupling strength of the system is further increased. Unfortunately, PIMC simulations are not feasible at these conditions due to the fermion sign prob-

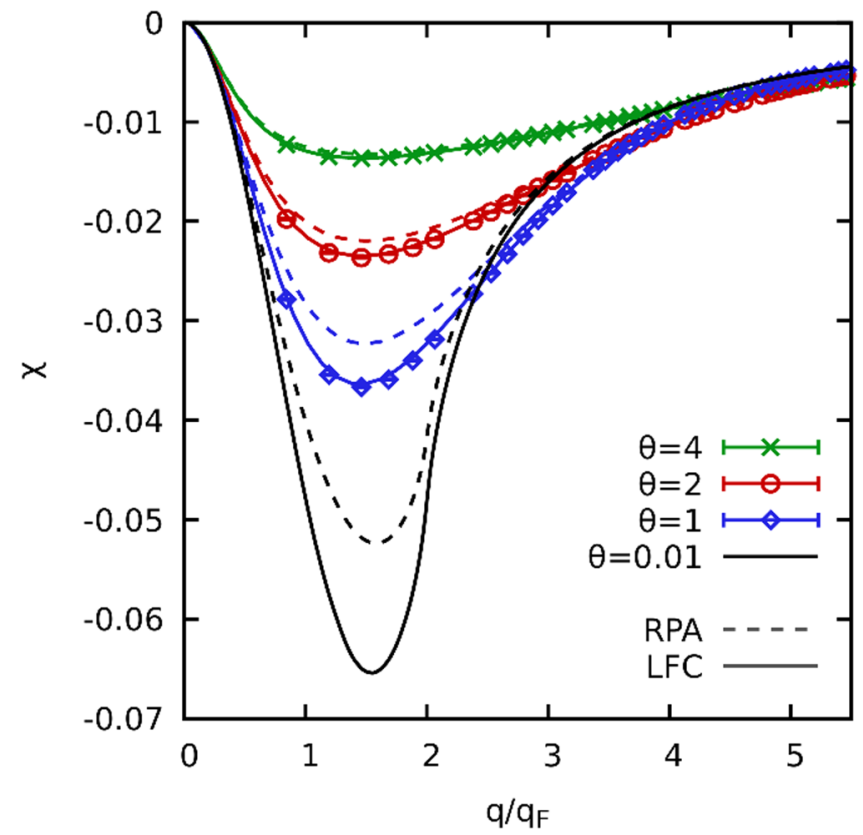

FIG. 17. Wave-number dependence of the linear response function $\chi^{(1)}(q)$ for the warm dense electron gas at $r_{s}=2$ and $\theta=1$ (blue diamonds), $\theta=2$ (red circles), and $\theta=4$ (green crosses). The dashed and solid lines have been obtained within RPA and using the LFC from Ref. [58], see Eq. (5). 

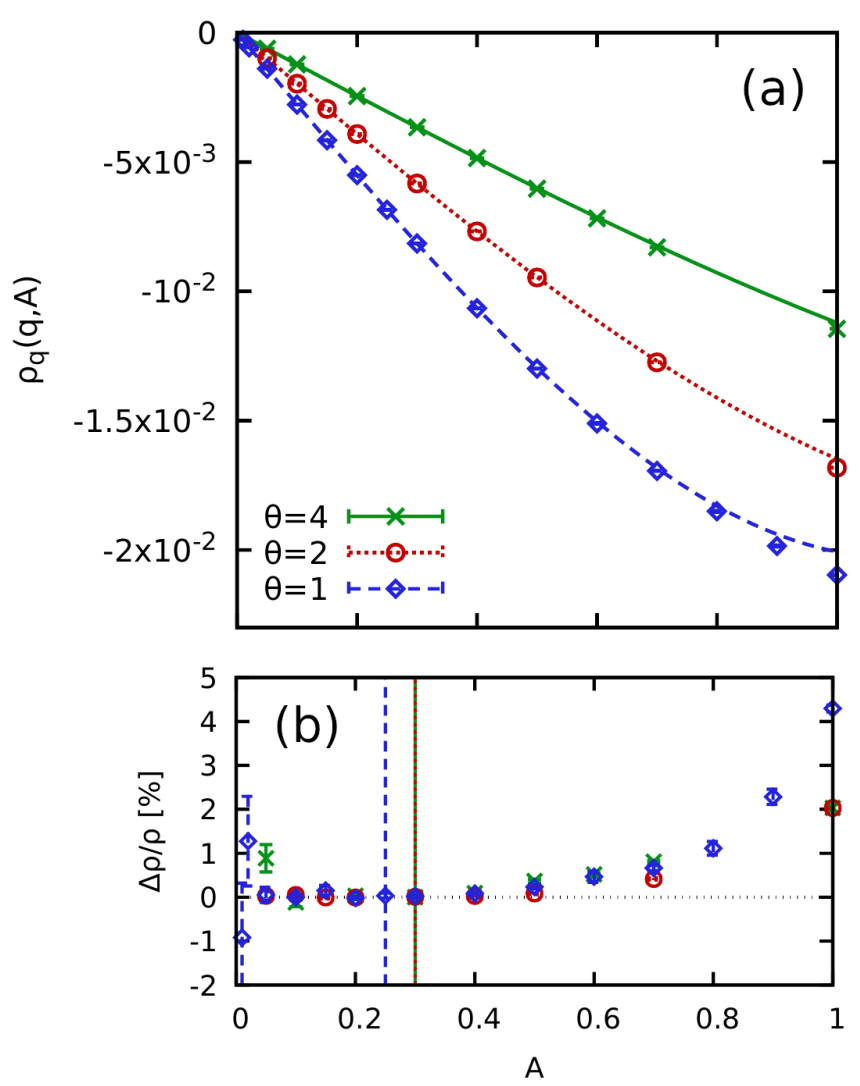

FIG. 18. Determination of the cubic response function $\chi^{(1, \text { cubic })}(q)$. Panel (a): Density response of the first harmonic $\left\langle\rho_{\mathbf{q}}\right\rangle_{q, A}$ of the UEG for $N=14, r_{s}=2$, and $q \approx 0.84 q_{\mathrm{F}}$ for $\theta=1$ (blue diamonds) $\theta=2$ (red circles), and $\theta=4$ (green crosses). The corresponding curves of the same color have been obtained by fitting Eq. (36) to the PIMC data. Panel (b): Relative deviation of the fits to the PIMC data. The vertical lines show the respective maximum value of $A$ that was included in the fits.

lem [64], and ground-state QMC simulations are not available for this quantity.

As a reference, we show the analogous temperature dependence of the LRT response function $\chi^{(1)}(q)$ in Fig. 17. Overall, $\chi^{(1)}(q)$ exhibits a qualitatively similar behavior as $\chi^{(2)}(q)$, with a screening-induced decay at small wave numbers, and a maximum of its modulus around $q \sim 1.5 q_{\mathrm{F}}$. Still, the decrease of the density response towards high temperature is substantially less pronounced for $\chi^{(1)}(q)$, which makes the experimental probing of the second harmonic a potentially valuable method of diagnostic for the temperature $[45,46]$.

The next generalized response function of interest is given by $\chi^{(1, \text { cubic })}(q)$, the determination of which is demonstrated in Fig. 18 for the usual three values of $\theta$. The data points are PIMC results for $\left\langle\hat{\rho}_{\mathbf{q}}\right\rangle_{q, A}$ and the different curves have been fitted to the latter via Eq. (36). Evidently, this functional form is again well suited to represent the PIMC data, and deviations can only be spotted with the naked eye at large perturbation amplitudes and are most pronounced for $\theta=1$. In panel (b), we show the corresponding relative deviations $\Delta \rho / \rho$, with the vertical lines representing the maximum values of the perturbation amplitude $A$ that have been included into the fitting procedure. In particular, the fit function remains accurate for

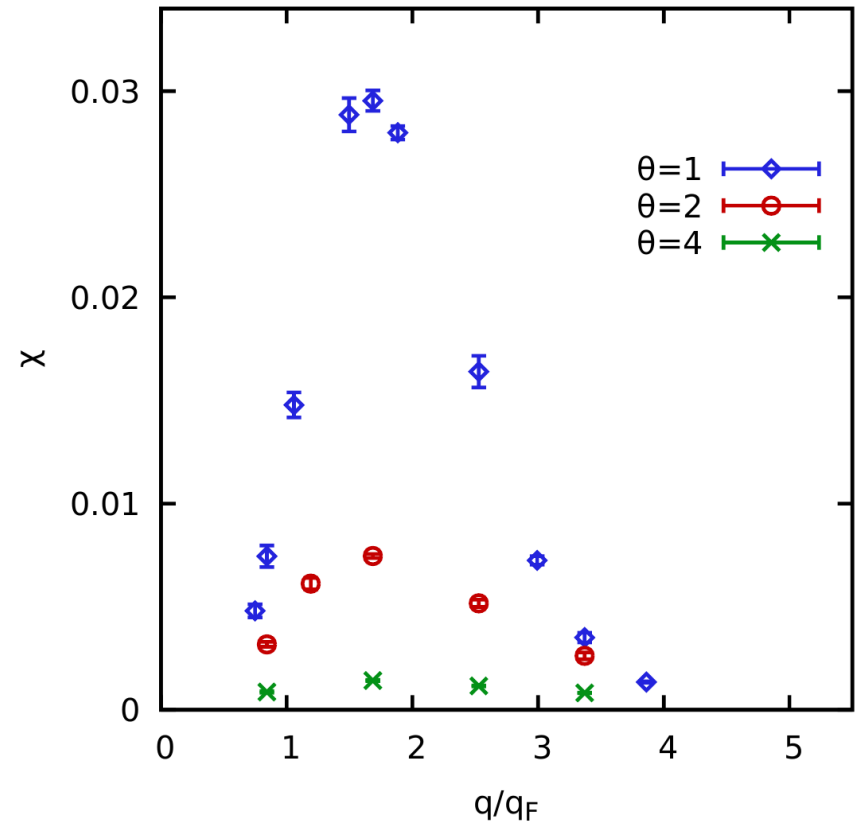

FIG. 19. Wave-number dependence of the cubic response function of the first harmonic $\chi^{(1, \text { cubic })}(q)$ for the warm dense electron gas at $r_{s}=2$ and $\theta=1$ (blue diamonds), $\theta=2$ (red circles), and $\theta=4$ (green crosses).

large values of $A$ beyond the respective fitting interval for all three temperatures, which is a strong indication for the high quality of our approach.

The full wave-number dependence of the thus determined cubic response function of the first harmonic $\chi^{(1, \text { cubic })}(q)$ is shown in Fig. 19. First and foremost, we find that this function exhibits an even more pronounced dependence on the temperature compared to both $\chi^{(1)}(q)$ and $\chi^{(2)}(q)$, which further highlights the potential utility of the nonlinear density response as a method of diagnostics for WDM. More specifically, the magnitude of the maximum at $\theta=4$ is more than order of magnitude smaller compared to its analog at $\theta=1$. Unfortunately, no RPA and LFC data are available in this case, as the ideal cubic response function is not known analytically and, hence, would require extensive PIMC simulations of the harmonically perturbed ideal Fermi gas.

The final response function considered in this work is given by the cubic response function of the third harmonic $\chi^{(3)}(q)$. The determination of this function on the basis of our PIMC data for $\left\langle\hat{\rho}_{3 \mathbf{q}}\right\rangle_{q, A}$ is shown in Fig. 20, where the curves have been fitted to the latter via Eq. (38). Since the observed signal for the third harmonic is comparably quite small for all three temperatures (cf. Fig. 14), the relative statistical uncertainty of the PIMC data points is large, especially at small $A$. Yet, Eq. (38) only constitutes the lowest order in $A$ of $\left\langle\hat{\rho}_{3 \mathbf{q}}\right\rangle_{q, A}$, which makes the determination of the coefficient $\chi^{(3)}(q)$ difficult. This is particularly true for $\theta=4$, where we can only give a qualitative estimation of the order of magnitude of this function.

The full wave-number dependence of $\chi^{(3)}(q)$ is shown in Fig. 21, again for a gamut of different values of $\theta$. For the highest temperature $(\theta=4$, green curve), the signal is two 


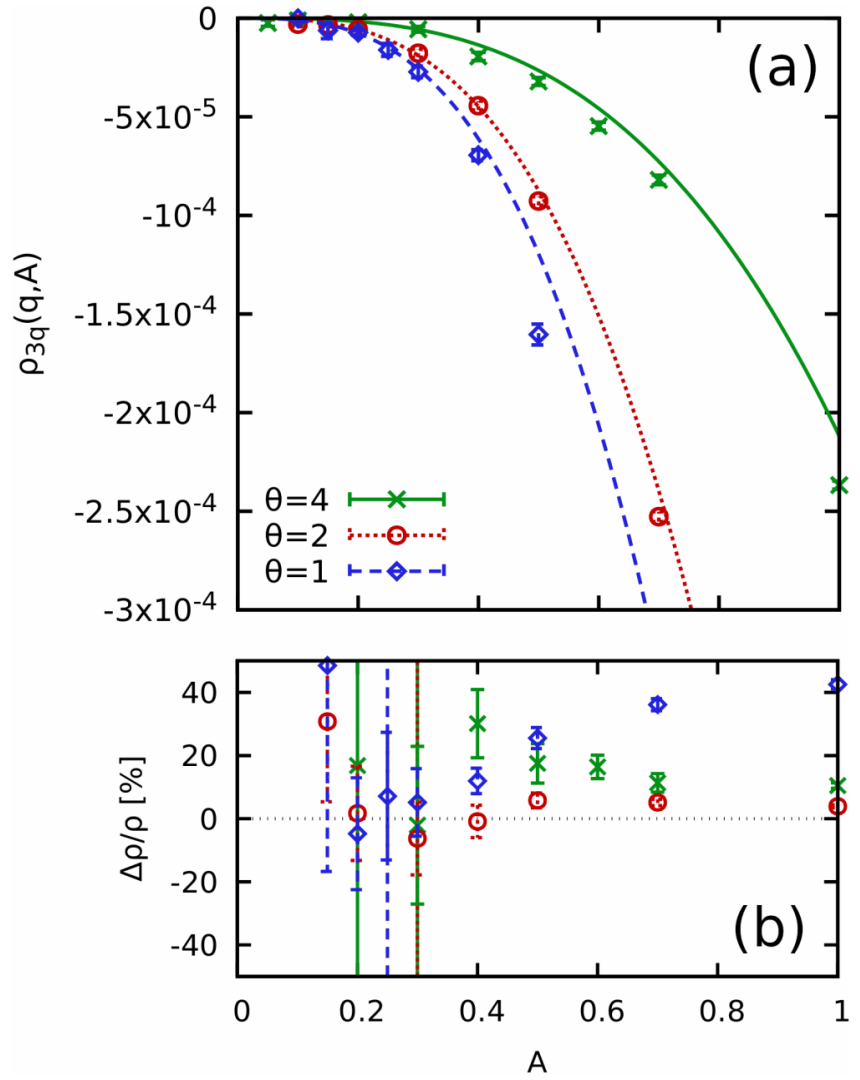

FIG. 20. Determination of the cubic response function $\chi^{(3)}(q)$. Panel (a): Density response of the third harmonic $\left\langle\rho_{3 \mathbf{q}}\right\rangle_{q, A}$ of the UEG for $N=14, r_{s}=2$, and $q \approx 0.84 q_{\mathrm{F}}$ for $\theta=1$ (blue diamonds) $\theta=2$ (red circles), and $\theta=4$ (green crosses). The corresponding curves of the same color have been obtained by fitting Eq. (37) to the PIMC data. Panel (b): Relative deviation of the fits from the PIMC data. The vertical lines show the respective maximum value of $A$ that was included in the fits.

orders of magnitude smaller than in the ground state $(\theta=$ 0.01 , black curve) and can hardly be resolved with the bare eye. Upon decreasing the temperature to $\theta=2$ (red curve), the signal is increased by a factor of five and can be resolved with the PIMC procedure described in the discussion of Fig. 20. Moreover, the difference between RPA [Eq. (30)] and the LFC-based description [Eq. (34)] is quite small, and the PIMC data points are in excellent agreement with the latter.

Decreasing the temperature by an additional factor of two leads to the blue curves and data points, which display approximately thrice the magnitude in the maximum of the density response around $q \sim 1.5 q_{\mathrm{F}}$. In addition, there appears a much larger difference between RPA and LFC, and-although to a much smaller degree-between the LFC curve and the PIMC data; see also the discussion of Fig. 6 above.

Lastly, the black curves correspond to the ground state $(\theta=0.01)$, where no PIMC simulations are available due to the fermion sign problem [64]. Evidently, RPA underestimates the depth of the minimum of $\chi^{(3)}(q)$ around $q \approx 1.5 q_{\mathrm{F}}$ by almost $50 \%$ and, thus, only gives a very qualitative description of the density response at the third harmonic of the original perturbation. In addition, we find a complicated, nontrivial behavior as $\chi^{(3)}(q)$ changes its sign around the Fermi wave

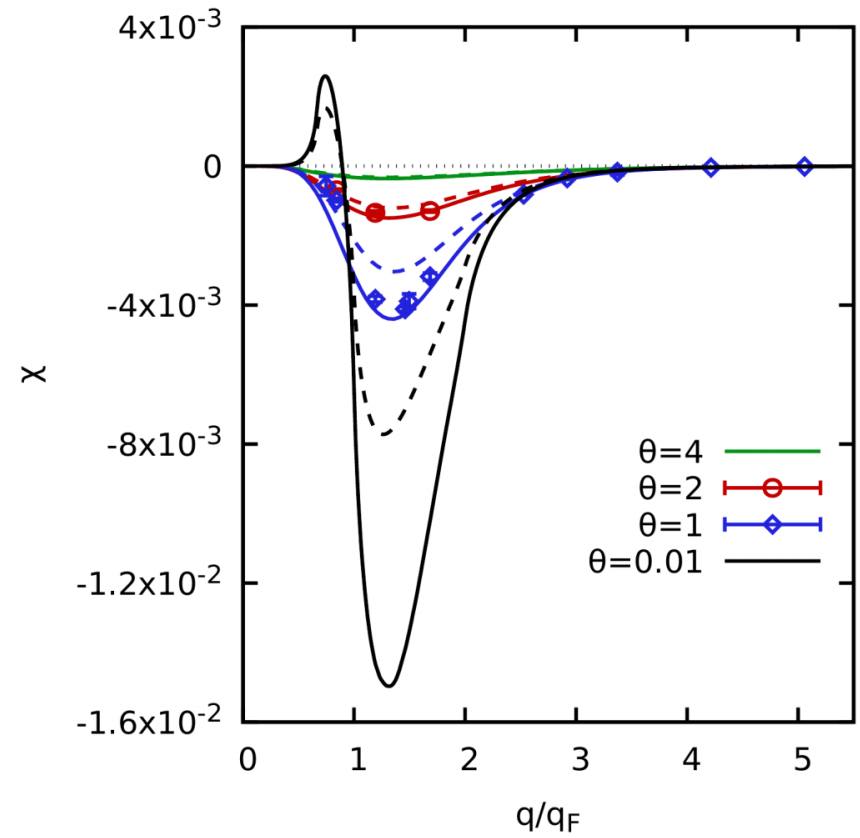

FIG. 21. Wave-number dependence of the cubic response function of the third harmonic $\chi^{(3)}(q)$ for the warm dense electron gas at $r_{s}=2$ and $\theta=1$ (blue diamonds), $\theta=2$ (red circles), and $\theta=4$ (green crosses). The solid and dashed lines have been obtained using the LFC from Ref. [58] and within RPA, respectively.

number. We expect this to be a real physical effect, as a quite similar behavior has been detected on the basis of our PIMC data at a lower density, $r_{s}=6$, shown in Fig. 22 below.

\section{Strong coupling: $r_{s}=6$}

Let us next investigate the nonlinear density response of the UEG at stronger coupling, $r_{s}=6$ and $\theta=1$. Such conditions can be realized experimentally for example in evaporation experiments [114-117] and constitute a challenging benchmark for models and theories due to the pronounced impact of electronic exchange-correlation effects [76,116].

Since the determination of the different generalized response functions from $a b$ initio PIMC data for $\left\langle\hat{\rho}_{\mathbf{k}}\right\rangle_{q, A}$ is completely analogous to the case of $r_{s}=2$ that was discussed in the previous sections, here we restrict ourselves to a concise discussion of the final results summarized in Fig. 22.

Panel (a) corresponds to the usual LRT function $\chi^{(1)}(q)$, where the green stars have been obtained from a single simulation of the unperturbed UEG by evaluating Eq. (6) for $N=34$ unpolarized electrons. These data are in good agreement with the dotted red curve that has been computed from the static LFC presented in Ref. [58], whereas RPA (dashed blue) substantially underestimates the magnitude of the minimum of $\chi^{(1)}(q)$ around $q=2 q_{\mathrm{F}}$ by more than $25 \%$. This is of course expected and a direct consequence of the increased importance of electronic correlation effects at low density [36]. The solid yellow curve depicts results for the ideal Fermi gas and becomes increasingly inaccurate towards small wave numbers, when screening effects start to manifest in the UEG [118]. 

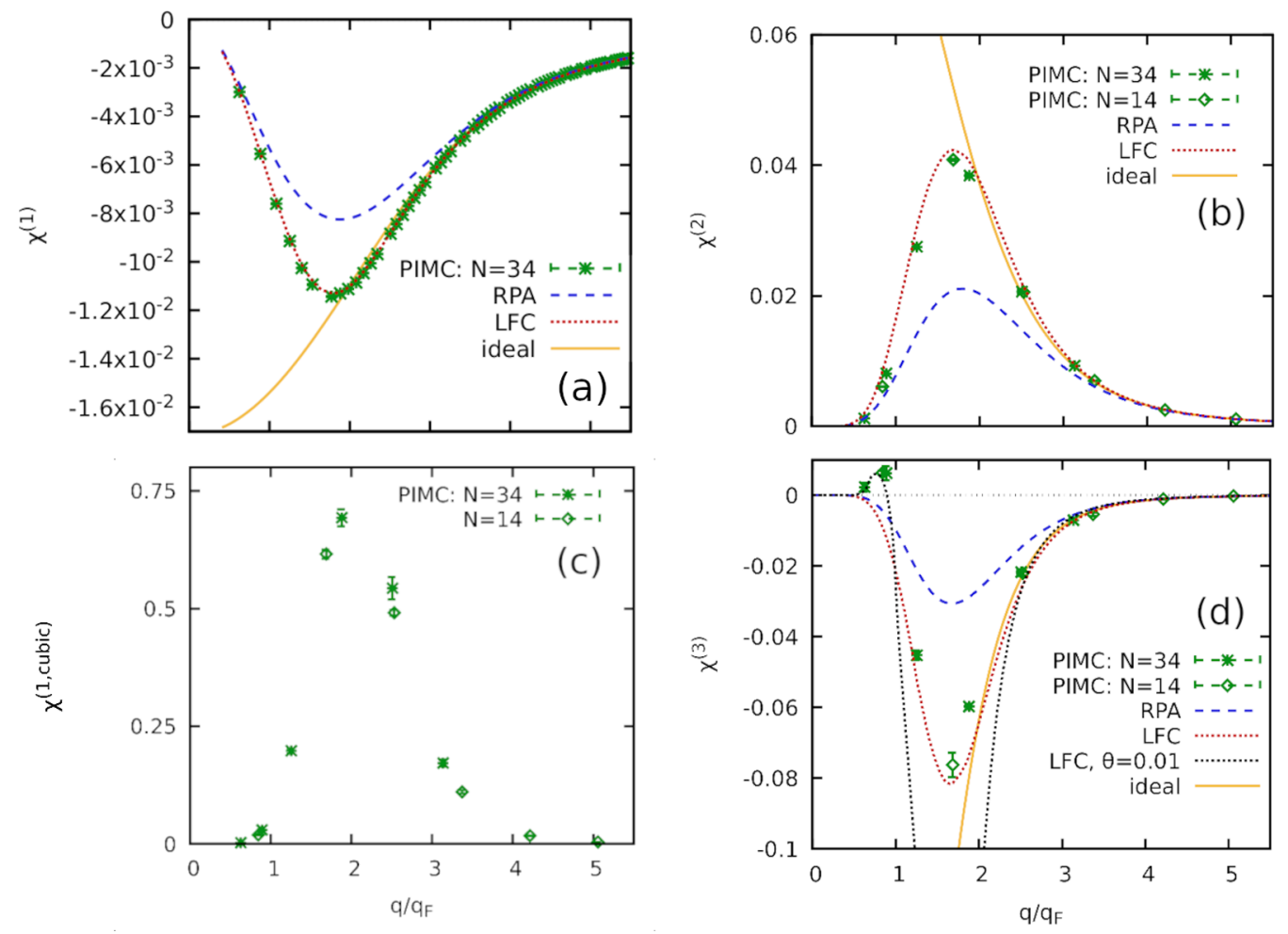

FIG. 22. Wave-number dependence of the linear response function $\chi^{(1)}(q)$ (a), the quadratic response function of the second harmonic $\chi^{(2)}(q)(\mathrm{b})$, the cubic response function of the first harmonic $\chi^{(1, \text { cubic) }}(q)(\mathrm{c})$, and the cubic response function of the third harmonic $\chi^{(3)}(q)$ (d). The green stars and diamonds correspond to PIMC data points for $N=34$ and $N=14$, the solid-yellow curve to the ideal Fermi gas, the dashed-blue curves to RPA, and the dotted-red curves have been obtained using the LFC from Ref. [58]. All data have been obtained for $r_{s}=6$ and $\theta=1$.

Panel (b) shows the wave-number dependence of the quadratic response function of the second harmonic, $\chi^{(2)}(q)$. We find that this function exhibits a significantly faster decay towards zero both in the small- and large- $q$ limits, similar to our findings for $r_{s}=2$ above. In addition, the electronic LFC $G(q)$ has a substantially larger impact on $\chi^{(2)}(q)$ compared to $\chi^{(1)}(q)$, and the RPA underestimates the peak at $q \approx 1.75 q_{\mathrm{F}}$ by $50 \%$. In contrast, the LFC curve is in good agreement with the PIMC data points for both $N=14$ (green diamonds) and $N=34$ (green stars) over the entire depicted wave-number range, and the deviation due to the approximate treatment of screening effects [cf. Sec. II] is small. In addition, the PIMC data points for the two system sizes exhibit a smooth progression, which means that no finite-size effects can be resolved within the given level of accuracy. This is in good agreement with the recent investigation of $\chi^{(1, \text { cubic })}(q)$ presented in Ref. [56].

Let us next focus on panel (c) showing the cubic density response function of the first harmonic, $\chi^{(1, \text { cubic })}(q)$ at the same conditions. In particular, this function is sharply peaked around $q \approx 2 q_{\mathrm{F}}$ and quickly vanishes both in the limits of small and large wave numbers. We again mention that the evaluation of the RPA and LFC expressions given in Eqs. (31) and (35) is not possible as $\chi_{0}^{(1, \text { cubic })}(q)$ cannot be readily evaluated.

Finally, we investigate the cubic density response function of the third harmonic, $\chi^{(3)}(q)$ shown in Fig. 22(d). While the associated density response at the third harmonic is relatively small and unimportant compared to the signals at $\mathbf{k}=\mathbf{q}$ and $\mathbf{k}=2 \mathbf{q}$, this function still deserves close attention as it exhibits interesting, nontrivial behavior. More specifically, the PIMC data points are positive for small wave numbers and $\chi^{(2)}(q)$ changes its sign around the Fermi wave number $q=q_{\mathrm{F}}$.

This trend is further substantiated in Fig. 23, where we show the dependence of the actual density response at the third harmonic $\left\langle\hat{\rho}_{3 \mathbf{q}}\right\rangle_{q, A}$ on the perturbation amplitude $A$. The green crosses show results for the smallest wave number accessible for $N=34$ electrons, $q \approx 0.62 q_{\mathrm{F}}$, and we can clearly resolve a positive density response that monotonically increases with $A$. In contrast, the blue diamonds and red circles corresponding to two larger wave numbers $\left(q \approx 1.25 q_{\mathrm{F}}\right.$ and $q \approx 2.51 q_{\mathrm{F}}$, respectively) both exhibit a negative density response. As usual, the different curves have been obtained by fitting Eq. (38) to the PIMC data, and Fig. 23(b) shows the relative deviation. Interestingly, the fit function remains accurate for larger $A$ at both $q=0.62 q_{\mathrm{F}}$ and $q=1.25 q_{\mathrm{F}}$ than for $q=2.51 q_{\mathrm{F}}$, even though the response is substantially larger at the intermediate wave number (see also the vertical lines in the bottom panel showing the maximum value of $A$ that has been included into the fitting procedure).

Let us get back to the full wave-number dependence of $\chi^{(3)}(q)$ shown in Fig. 22(d). Evidently, neglecting electronic exchange-correlation effects within the RPA (dashed-blue 


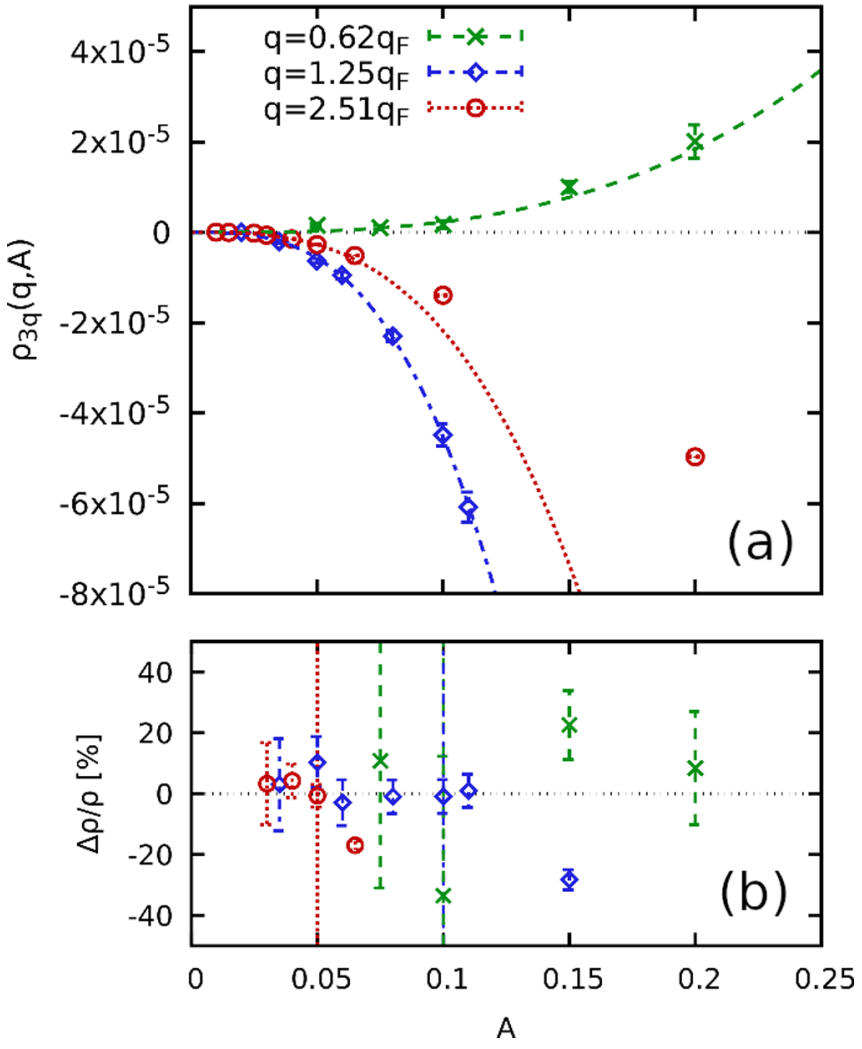

FIG. 23. Determination of the cubic response function $\chi^{(3)}(q)$. Panel (a): Density response of the third harmonic $\left\langle\hat{\rho}_{3 \mathbf{q}}\right\rangle_{q, A}$ of the UEG for $N=34, r_{s}=6$, and $\theta=1$ for $q \approx 0.62 q_{\mathrm{F}}$ (green crosses) $q \approx 1.25 q_{\mathrm{F}}$ (blue diamonds), and $q \approx 2.51 q_{\mathrm{F}}$ (red circles). The corresponding curves of the same color have been obtained by fitting Eq. (38) to the PIMC data. Panel (b): Relative deviation of the fits to the PIMC data. The vertical lines show the respective maximum value of $A$ that was included in the fits.

curve) results in a very poor description of the cubic response function, and the real depth of the minimum is underestimated by a factor of two. Including the static LFC gives the dotted red curve that is in very good agreement with the PIMC data points for $q \gtrsim q_{\mathrm{F}}$. Yet, the LFC result, Eq. (34), is not capable to describe the sign change in $\chi^{(3)}(q)$ around the Fermi wave number and, thus, stays negative over the entire $q$ range. This, in turn, means that the approximate linear treatment of screening effects [cf. Sec. II above] leads to a description where this sign change is missed.

Finally, the dotted black curve has also been obtained by evaluating Eq. (34) including the LFC, but for $\theta=0.01$, i.e, in the ground state. While this leads to a curve with a substantially deeper minimum around $q \sim 1.5 q_{\mathrm{F}}$ than at the Fermi temperature, it does exhibit the sign change in $\chi^{(3)}(q)$ that we find in our PIMC data at the higher temperature.

\section{SUMMARY AND DISCUSSION}

In this paper, we have presented an in-depth analysis of nonlinear effects in the density response of the warm dense electron gas, substantially extending the first investigation of Ref. [56]. We have obtained extensive PIMC results carrying out simulations of a harmonically perturbed electron gas, which has allowed us to obtain the full spectrum of excitations at the integer harmonics of the original perturbation. First and foremost, we have found that the dominant nonlinear contribution is given by the quadratic response at the second harmonic, for weak to moderate values of the perturbation amplitude $A$. The second potentially important nonlinear term is given by the cubic response at the first harmonic studied in Ref. [56], whereas the cubic response at the third harmonic is practically negligible in most realistic situations. In addition, we have found that the nonlinear response functions more strongly depend on system parameters such as the temperature compared to the usual LRT, which makes them a potentially valuable tool of diagnostics for WDM experiments. In addition, the nonlinear response is strongly shaped by electronic exchange-correlation effects, which makes a reliable theory based on $a b$ initio PIMC data indispensable. Furthermore, we have extended previous works on the nonlinear density response of warm dense electrons and presented expressions for the response functions in terms of the well-known LFC that is readily available based on previous PIMC simulations $[58,59,76]$. In particular, this approach is capable to reproduce the benchmark PIMC data for the nonlinear density response with negligible computational cost.

It is important to note that the present paper has been limited to the nonlinear density response of the UEG, which is a highly important, yet substantially simplified model system $[36,55]$. Consequently, the response of a real physical system that is composed of both electrons and ions is expected to be substantially more complicated. For example, the excitation of atoms for wave numbers in the vicinity of $q_{\mathrm{F}}$ cannot be addressed within the framework of the UEG. Still, we stress that the density response of the UEG constitutes the starting point for many applications in WDM theory and beyond, often with reasonable accuracy [1]. Therefore, our new results can directly be used to take into account nonlinear effects in the description of properties such as the stopping power or electronically screened potentials. Moreover, the UEG constitutes a remarkably good approximation for the conduction electrons of metals such as aluminum [76]. This is particularly true at warm dense matter conditions, when electron-ion coupling is weak. Lastly, we mention that the accurate description of the nonlinear density response of the UEG is interesting in its own right as it further advances our current understanding of this fundamental model system [36,55,119].

In addition, we mention that intense laser interaction with warm dense matter gives rise to various nonequilibrium effects that have been studied, e.g., with quantum kinetic equations $[13,120]$. The first is due to scattering in the presence of the field. Note that electron-electron scattering is not modified by the laser field (two particles move in phase) and quickly establishes an equilibrium distribution. On the other hand, electron-ion scattering is strongly modified giving rise to inverse bremsstrahlung heating of the electrons [120] slowly increasing the temperature of the equilibrium electrons. The second effect is a modification of the electronic spectral function, which develops photonic side peaks [13]. In principle, both effects can be qualitatively captured by PIMC simulations, which will be studied elsewhere.

While the present analysis was based on simulations for a purely static density perturbation, even stronger nonlinear 
effects are to be expected for dynamic excitations. For example, the excitation of warm dense matter by a strong laser field will give rise to a time-dependent density response that will involve harmonics. If proper resonance conditions are fulfilled, as e.g., in the case of high harmonics generation in rare gases [9], or laser plasmas [120], the excitation of even higher harmonic orders should be expected.

We are convinced that the findings of our paper will open up many avenues of future research in multiple directions. For example, a realistic perturbation in a WDM experiment will be given by a superposition of many distinct harmonic perturbations. While this has no profound consequence within LRT, a nonlinear treatment of the density response will give rise to mode-coupling between different perturbations, which will make the excitation spectrum more interesting and complicated. One can imagine two-color x-ray beam experiments to intentionally use the mode coupling for diagnostics. A second topic for future research is given by the investigation of the dynamic density response, which can be done on the basis of our new theoretical expressions and a suitable local field correction such as the ESA presented in Refs. $[59,76]$. In addition to these basic questions, we stress that an accurate theory of nonlinear effects can be directly used for the improved computation of different material properties such as the electronic stopping power, screened potentials, or en- ergy relaxation rates. Lastly, the investigation of the nonlinear density response of real physical systems such as warm dense hydrogen based on either $a b$ initio PIMC simulations or more approximate density functional theory constitutes a challenging and highly interesting project and will help to resolve the as of yet poorly understood interplay between the electrons and ions in this regime.

\section{ACKNOWLEDGMENTS}

This work was partly funded by the Center for Advanced Systems Understanding (CASUS) which is financed by Germany's Federal Ministry of Education and Research (BMBF) and by the Saxon Ministry for Science, Culture and Tourism (SMWK) with tax funds on the basis of the budget approved by the Saxon State Parliament, and by the Deutsche Forschungsgemeinschaft (DFG) via Project BO1366/15. The PIMC calculations were carried out at the Norddeutscher Verbund für Hoch- und Höchstleistungsrechnen (HLRN) under Grant shp00026, on a Bull Cluster at the Center for Information Services and High Performace Computing (ZIH) at Technische Universität Dresden, on the cluster hemera at Helmholtz-Zentrum Dresden-Rossendorf (HZDR), and at the computing center (Rechenzentrum) of Kiel university.
[1] G. Giuliani and G. Vignale, Quantum Theory of the Electron Liquid (Cambridge University Press, Cambridge, 2008).

[2] H. Haug and A.-P. Jauho, Quantum Kinetics in Transport and Optics of Semiconductors (Springer, Heidelberg, 2008).

[3] N. H. Kwong, M. Bonitz, R. Binder, and H. S. Köhler, Semiconductor Kadanoff-Baym equation results for optically excited electron-hole plasmas in quantum wells, Phys. Status Solidi B 206, 197 (1998).

[4] D. M. Ceperley, Path integrals in the theory of condensed helium, Rev. Mod. Phys 67, 279 (1995).

[5] Yu Shi, Superfluidity or supersolidity as a consequence of off-diagonal long-range order, Phys. Rev. B 72, 014533 (2005).

[6] S. Y. Savrasov and D. Y. Savrasov, Electron-phonon interactions and related physical properties of metals from linear-response theory, Phys. Rev. B 54, 16487 (1996).

[7] E. K. U. Gross and Walter Kohn, Local Density-Functional Theory of Frequency-Dependent Linear Response, Phys. Rev. Lett. 55, 2850 (1985).

[8] L. V. Keldysh, Ionization in the field of a strong electromagnetic wave, JETP 20, 1307 (1965).

[9] K. J. Schafer, B. Yang, L. F. DiMauro, and K. C. Kulander, Above Threshold Ionization Beyond the High Harmonic Cutoff, Phys. Rev. Lett. 70, 1599 (1993).

[10] N. Bloembergen, Nonlinear Optics, 4th ed. (World Scientific, Singapore, 1996).

[11] F. Sakata, Nonlinear science in nuclear physics, in Frontiers of Theoretical Physics (World Scientific, Singapore, 2001), p. 35.

[12] P. Sprangle, E. Esarey, and A. Ting, Nonlinear Theory of Intense Laser-Plasma Interactions, Phys. Rev. Lett. 64, 2011 (1990).
[13] D. Kremp, Th. Bornath, M. Bonitz, and M. Schlanges, Quantum kinetic theory of plasmas in strong laser fields, Phys. Rev. E 60, 4725 (1999).

[14] M. Bonitz, Th. Bornath, D. Kremp, M. Schlanges, and W. D. Kraeft, Quantum kinetic theory for laser plasmas. dynamical screening in strong fields, Contrib. Plasma Phys. 39, 329 (1999).

[15] Ira B. Bernstein, Waves in a plasma in a magnetic field, Phys. Rev. 109, 10 (1958).

[16] M. Bonitz, Z. Donkó, T. Ott, H. Kählert, and P. Hartmann, Nonlinear Magnetoplasmons in Strongly Coupled Yukawa Plasmas, Phys. Rev. Lett. 105, 055002 (2010).

[17] T. Ott, M. Bonitz, P. Hartmann, and Z. Donkó, Higher harmonics of the magnetoplasmon in strongly coupled Coulomb and Yukawa systems, Phys. Rev. E 83, 046403 (2011).

[18] E. Hendry, P. J. Hale, J. Moger, A. K. Savchenko, and S. A. Mikhailov, Coherent Nonlinear Optical Response of Graphene, Phys. Rev. Lett. 105, 097401 (2010).

[19] S. A. Mikhailov, Nonlinear Electromagnetic Response of a Uniform Electron Gas, Phys. Rev. Lett. 113, 027405 (2014).

[20] Joel D. Cox, Iván Silveiro, and F. Javier García de Abajo, Quantum effects in the nonlinear response of graphene plasmons, ACS Nano 10, 1995 (2016).

[21] W. J. Blau, H. J. Byrne, D. J. Cardin, T. J. Dennis, J. P. Hare, H. W. Kroto, R. Taylor, and D. R. M. Walton, Large Infrared Nonlinear Optical Response of $\mathrm{c}_{60}$, Phys. Rev. Lett. 67, 1423 (1991).

[22] J. Lee, M. Tymchenko, C. Argyropoulos, P-Y. Chen, F. Lu, F. Demmerle, G. Boehm, M-C. Amann, A. Alù, and M. A. Belkin, Giant nonlinear response from plasmonic metasurfaces coupled to intersubband transitions, Nature (London) 511, 65 (2014). 
[23] F. Graziani, M. P. Desjarlais, R. Redmer, and S. B. Trickey, eds., Frontiers and Challenges in Warm Dense Matter (Springer, New York, 2014).

[24] V. E. Fortov, Extreme states of matter on earth and in space, Phys. Usp. 52, 615 (2009).

[25] M. Bonitz, T. Dornheim, Zh. A. Moldabekov, S. Zhang, P. Hamann, H. Kählert, A. Filinov, K. Ramakrishna, and J. Vorberger, Ab initio simulation of warm dense matter, Phys. Plasmas 27, 042710 (2020).

[26] B. Militzer, W. B. Hubbard, J. Vorberger, I. Tamblyn, and S. A. Bonev, A massive core in jupiter predicted from firstprinciples simulations, Astrophys. J. 688, L45 (2008).

[27] J. Vorberger, I. Tamblyn, B. Militzer, and S. A. Bonev, Hydrogen-helium mixtures in the interiors of giant planets, Phys. Rev. B 75, 024206 (2007).

[28] D. Saumon, W. B. Hubbard, G. Chabrier, and H. M. van Horn, The role of the molecular-metallic transition of hydrogen in the evolution of jupiter, saturn, and brown dwarfs, Astrophys. J 391, 827 (1992).

[29] A. Becker, W. Lorenzen, J. J. Fortney, N. Nettelmann, M. Schöttler, and R. Redmer, Ab initio equations of state for hydrogen (H-REOS.3) and helium (He-REOS.3) and their implications for the interior of brown dwarfs, Astrophys. J. Suppl. Ser 215, 21 (2014).

[30] S. X. Hu, B. Militzer, V. N. Goncharov, and S. Skupsky, First-principles equation-of-state table of deuterium for inertial confinement fusion applications, Phys. Rev. B 84, 224109 (2011).

[31] M. L. Brongersma, N. J. Halas, and P. Nordlander, Plasmoninduced hot carrier science and technology, Nat. Nanotechnol. 10, 25 (2015).

[32] D. Kraus, A. Ravasio, M. Gauthier, D. O. Gericke, J. Vorberger, S. Frydrych, J. Helfrich, L. B. Fletcher, G. Schaumann, B. Nagler et al., Nanosecond formation of diamond and lonsdaleite by shock compression of graphite, Nat. Commun. 7, 10970 (2016).

[33] D. Kraus, J. Vorberger, A. Pak, N. J. Hartley, L. B. Fletcher, S. Frydrych, E. Galtier, E. J. Gamboa, D. O. Gericke, S. H. Glenzer et al., Formation of diamonds in laser-compressed hydrocarbons at planetary interior conditions, Nat. Astron. 1, 606 (2017).

[34] K. Falk, Experimental methods for warm dense matter research, High Power Laser Sci. Eng 6, e59 (2018).

[35] D. Kremp, M. Schlanges, and W.-D. Kraeft, Quantum Statistics of Nonideal Plasmas (Springer, Heidelberg, 2005).

[36] T. Dornheim, S. Groth, and M. Bonitz, The uniform electron gas at warm dense matter conditions, Phys. Rep. 744, 1 (2018).

[37] T. Dornheim, S. Groth, F. D. Malone, T. Schoof, T. Sjostrom, W. M. C. Foulkes, and M. Bonitz, Ab initio quantum Monte Carlo simulation of the warm dense electron gas, Phys. Plasmas 24, 056303 (2017).

[38] K. P. Driver and B. Militzer, All-Electron Path Integral Monte Carlo Simulations of Warm Dense Matter: Application to Water and Carbon Plasmas, Phys. Rev. Lett. 108, 115502 (2012).

[39] E. W. Brown, B. K. Clark, J. L. DuBois, and D. M. Ceperley, Path-Integral Monte Carlo Simulation of the Warm Dense Homogeneous Electron Gas, Phys. Rev. Lett. 110, 146405 (2013).

[40] B. Militzer and K. P. Driver, Development of Path Integral Monte Carlo Simulations with Localized Nodal Surfaces for Second-Row Elements, Phys. Rev. Lett. 115, 176403 (2015).
[41] T. Dornheim, S. Groth, T. Sjostrom, F. D. Malone, W. M. C. Foulkes, and M. Bonitz, Ab Initio Quantum Monte Carlo Simulation of the Warm Dense Electron Gas in the Thermodynamic Limit, Phys. Rev. Lett. 117, 156403 (2016).

[42] T. Dornheim, S. Groth, J. Vorberger, and M. Bonitz, Ab initio Path Integral Monte Carlo Results for the Dynamic Structure Factor of Correlated Electrons: From the Electron Liquid to Warm Dense Matter, Phys. Rev. Lett. 121, 255001 (2018).

[43] F. D. Malone, N. S. Blunt, E. W. Brown, D. K. K. Lee, J. S. Spencer, W. M. C. Foulkes, and J. J. Shepherd, Accurate Exchange-Correlation Energies for the Warm Dense Electron Gas, Phys. Rev. Lett. 117, 115701 (2016).

[44] S. Groth, T. Dornheim, T. Sjostrom, F. D. Malone, W. M. C. Foulkes, and M. Bonitz, Ab initio Exchange-Correlation Free Energy of the Uniform Electron Gas at Warm Dense Matter Conditions, Phys. Rev. Lett. 119, 135001 (2017).

[45] S. H. Glenzer and R. Redmer, X-ray Thomson scattering in high energy density plasmas, Rev. Mod. Phys 81, 1625 (2009).

[46] D. Kraus, B. Bachmann, B. Barbrel, R. W. Falcone, L. B. Fletcher, S. Frydrych, E. J. Gamboa, M. Gauthier, D. O. Gericke, S. H. Glenzer et al., Characterizing the ionization potential depression in dense carbon plasmas with high-precision spectrally resolved x-ray scattering, Plasma Phys. Control Fusion 61, 014015 (2019).

[47] G. Senatore, S. Moroni, and D. M. Ceperley, Local field factor and effective potentials in liquid metals, J. Non-Cryst. Sol 205-207, 851 (1996).

[48] Zh. A. Moldabekov, S. Groth, T. Dornheim, H. Kählert, M. Bonitz, and T. S. Ramazanov, Structural characteristics of strongly coupled ions in a dense quantum plasma, Phys. Rev. E 98, 023207 (2018).

[49] Zh. A. Moldabekov, H. Kählert, T. Dornheim, S. Groth, M. Bonitz, and T. S. Ramazanov, Dynamical structure factor of strongly coupled ions in a dense quantum plasma, Phys. Rev. E 99, 053203 (2019).

[50] J. Vorberger, D. O. Gericke, Th. Bornath, and M. Schlanges, Energy relaxation in dense, strongly coupled two-temperature plasmas, Phys. Rev. E 81, 046404 (2010).

[51] L. X. Benedict, M. P. Surh, L. G. Stanton, C. R. Scullard, A. A. Correa, J. I. Castor, F. R. Graziani, L. A. Collins, O. Certík, J. D. Kress, and M. S. Murillo, Molecular dynamics studies of electron-ion temperature equilibration in hydrogen plasmas within the coupled-mode regime, Phys. Rev. E 95, 043202 (2017).

[52] L. Waldecker, R. Bertoni, R. Ernstorfer, and J. Vorberger, Electron-Phonon Coupling and Energy Flow in a Simple Metal Beyond the Two-Temperature Approximation, Phys. Rev. X 6, 021003 (2016).

[53] W. Cayzac, A. Frank, A. Ortner, V. Bagnoud, M. M. Basko, S. Bedacht, C. Bläser, A. Blazevic, S. Busold, O. Deppert et al., Experimental discrimination of ion stopping models near the Bragg peak in highly ionized matter, Nat. Commun. 8, 15693 (2017).

[54] Zh. A. Moldabekov, T. Dornheim, M. Bonitz, and T. S. Ramazanov, Ion energy-loss characteristics and friction in a free-electron gas at warm dense matter and nonideal dense plasma conditions, Phys. Rev. E 101, 053203 (2020).

[55] P.-F. Loos and P. M. W. Gill, The uniform electron gas, Comput. Mol. Sci 6, 410 (2016). 
[56] T. Dornheim, J. Vorberger, and M. Bonitz, Nonlinear Electronic Density Response in Warm Dense Matter, Phys. Rev. Lett. 125, 085001 (2020).

[57] Zh. A. Moldabekov, T. Dornheim, and A. Cangi, Thermal signals from collective electronic excitations in inhomogeneous warm dense matter, arXiv:2105.09721.

[58] T. Dornheim, J. Vorberger, S. Groth, N. Hoffmann, Zh. A. Moldabekov, and M. Bonitz, The static local field correction of the warm dense electron gas: An ab initio path integral Monte Carlo study and machine learning representation, J. Chem. Phys 151, 194104 (2019).

[59] T. Dornheim, Zh. A. Moldabekov, and P. Tolias, Analytical representation of the local field correction of the uniform electron gas within the effective static approximation, Phys. Rev. B 103, 165102 (2021).

[60] M. F. Herman, E. J. Bruskin, and B. J. Berne, On path integral Monte Carlo simulations, J. Chem. Phys. 76, 5150 (1982).

[61] M. Takahashi and M. Imada, Monte Carlo calculation of quantum systems, J. Phys. Soc. Jpn. 53, 963 (1984).

[62] N. Metropolis, A. W. Rosenbluth, M. N. Rosenbluth, A. H. Teller, and E. Teller, Equation of state calculations by fast computing machines, J. Chem. Phys. 21, 1087 (1953).

[63] M. Troyer and U. J. Wiese, Computational Complexity and Fundamental Limitations to Fermionic Quantum Monte Carlo Simulations, Phys. Rev. Lett. 94, 170201 (2005).

[64] T. Dornheim, Fermion sign problem in path integral Monte Carlo simulations: Quantum dots, ultracold atoms, and warm dense matter, Phys. Rev. E 100, 023307 (2019).

[65] D. M. Ceperley, Fermion nodes, J. Stat. Phys. 63, 1237 (1991).

[66] T. Schoof, S. Groth, J. Vorberger, and M. Bonitz, Ab Initio Thermodynamic Results for the Degenerate Electron Gas at Finite Temperature, Phys. Rev. Lett. 115, 130402 (2015).

[67] F. Mezzacapo and M. Boninsegni, Structure, superfluidity, and quantum melting of hydrogen clusters, Phys. Rev. A 75, 033201 (2007).

[68] M. Boninsegni, N. V. Prokofev, and B. V. Svistunov, Worm algorithm and diagrammatic Monte Carlo: A new approach to continuous-space path integral Monte Carlo simulations, Phys. Rev. E 74, 036701 (2006).

[69] M. Boninsegni, N. V. Prokof'ev, and B. Svistunov, Worm Algorithm for Continuous-Space Path Integral Monte Carlo Simulations, Phys. Rev. Lett. 96, 070601 (2006).

[70] C. Bowen, G. Sugiyama, and B. J. Alder, Static dielectric response of the electron gas, Phys. Rev. B 50, 14838 (1994).

[71] S. Moroni, D. M. Ceperley, and G. Senatore, Static response from quantum Monte Carlo calculations, Phys. Rev. Lett. 69, 1837 (1992).

[72] S. Moroni, D. M. Ceperley, and G. Senatore, Static Response and Local Field Factor of the Electron Gas, Phys. Rev. Lett. 75, 689 (1995).

[73] S. Groth, T. Dornheim, and M. Bonitz, Configuration path integral Monte Carlo approach to the static density response of the warm dense electron gas, J. Chem. Phys 147, 164108 (2017).

[74] T. Dornheim, S. Groth, J. Vorberger, and M. Bonitz, Permutation blocking path integral Monte Carlo approach to the static density response of the warm dense electron gas, Phys. Rev. E 96, 023203 (2017).
[75] L. Mühlbacher and E. Rabani, Real-Time Path Integral Approach to Nonequilibrium Many-Body Quantum Systems, Phys. Rev. Lett. 100, 176403 (2008).

[76] T. Dornheim, A. Cangi, K. Ramakrishna, M. Böhme, S.i Tanaka, and J. Vorberger, Effective Static Approximation: A Fast and Reliable Tool for Warm-Dense Matter Theory, Phys. Rev. Lett. 125, 235001 (2020).

[77] S. Groth, T. Dornheim, and J. Vorberger, Ab initio path integral Monte Carlo approach to the static and dynamic density response of the uniform electron gas, Phys. Rev. B 99, 235122 (2019).

[78] T. Dornheim and J. Vorberger, Finite-size effects in the reconstruction of dynamic properties from ab initio path integral Monte Carlo simulations, Phys. Rev. E 102, 063301 (2020).

[79] Y. Kora and M. Boninsegni, Dynamic structure factor of superfluid ${ }^{4} \mathrm{He}$ from quantum Monte Carlo: Maximum entropy revisited, Phys. Rev. B 98, 134509 (2018).

[80] E. Vitali, M. Rossi, L. Reatto, and D. E. Galli, Ab initio lowenergy dynamics of superfluid and solid ${ }^{4} \mathrm{He}$, Phys. Rev. B 82, 174510 (2010).

[81] S. Saccani, S. Moroni, and M. Boninsegni, Excitation Spectrum of a Supersolid, Phys. Rev. Lett. 108, 175301 (2012).

[82] M. Boninsegni and D. M. Ceperley, Density fluctuations in liquid $4 \mathrm{He}$. Path integrals and maximum entropy, J. Low Temp. Phys. 104, 339 (1996).

[83] M. Motta, D. E. Galli, S. Moroni, and E. Vitali, Imaginary time density-density correlations for two-dimensional electron gases at high density, J. Chem. Phys. 143, 164108 (2015).

[84] D. C. Langreth, Linear and nonlinear response theory with applications, in Linear and Nonlinear Electron Transport in Solids, edited by J. T. Devreese and V. E. van Doren (Springer, Boston, 1976), p. 3.

[85] K. I. Golden, F. Green, and D. Neilson, Nonlinear-responsefunction approach to binary ionic mixtures: Dynamical theory, Phys. Rev. A 32, 1669 (1985).

[86] M. Bonitz, D. C. Scott, R. Binder, and S. W. Koch, Nonlinear carrier-plasmon interaction in a one-dimensional quantum plasma, Phys. Rev. B 50, 15095 (1994).

[87] N.-H. Kwong and M. Bonitz, Real-Time Kadanoff-Baym Approach to Plasma Oscillations in a Correlated Electron Gas, Phys. Rev. Lett 84, 1768 (2000).

[88] C. D. Hu and E. Zaremba, $Z^{3}$ correction to the stopping power of ions in an electron gas, Phys. Rev. B 37, 9268 (1988).

[89] A. Bergara, J. M. Pitarke, and P. M. Echenique, Quadratic electronic response of a two-dimensional electron gas, Phys. Rev. B 59, 10145 (1999).

[90] R. Cenni and P. Saracco, Evaluation of a class of diagrams useful in many-body calculations, Nucl. Phys. A 487, 279 (1988).

[91] A. Bergara, I. Campillo, J. M. Pitarke, and P. M. Echenique, Quadratic induced polarization by an external heavy charge in an electron gas, Phys. Rev. B 56, 15654 (1997).

[92] G. J. Kalman and J. M. Rommel, Theory of quadratic response, J. Phys. IV France 10, Pr5-161 (2000).

[93] G. Paasch and P. Rennert, Quadratic electronic polarizability of the interacting electron gas, Phys. Status Solidi B 83, 501 (1977).

[94] G. Paasch and A. Heinrich, Quadratic response of the electron gas. inclusion of local field corrections, Phys. Status Solidi B 102, 323 (1980). 
[95] J. M. Pitarke, R. H. Ritchie, and P. M. Echenique, Quadratic response theory of the energy loss of charged particles in an electron gas, Phys. Rev. B 52, 13883 (1995).

[96] J. M. Rommel and G. Kalman, Analytical properties of the quadratic density response and quadratic dynamical structure functions: Conservation sum rules and frequency moments, Phys. Rev. E 54, 3518 (1996).

[97] K. I. Golden, G. Kalman, and T. Datta, Sum rules for nonlinear plasma response functions, Phys. Rev. A 11, 2147 (1975).

[98] K. I. Golden and G. Kalman, Quadratic compressibility sum-rule test for strongly coupled one-component-plasma approximation schemes, Phys. Rev. A 17, 390 (1978).

[99] S.A. Mikhailov, Second-order response of a uniform threedimensional electron gas to a longitudinal electric field, Ann. Phys. (NY) 524, 182 (2012).

[100] H. Rostami, M. I. Katsnelson, and M. Polini, Theory of plasmonic effects in nonlinear optics: The case of graphene, Phys. Rev. B 95, 035416 (2017).

[101] J. Vorberger, Z.A. Moldabekov, M. Bonitz, and T. Dornheim, Theory of plasmonic effects in nonlinear optics: The case of graphene (unpublished).

[102] P. Hamann, T. Dornheim, J. Vorberger, Z. Moldabekov, and M. Bonitz, Dynamic Properties of the warm dense Electron gas: An ab initio path integral Monte Carlo approach (unpublished).

[103] P. Sperling, E. J. Gamboa, H. J. Lee, H. K. Chung, E. Galtier, Y. Omarbakiyeva, H. Reinholz, G. Röpke, U. Zastrau, J. Hastings, L. B. Fletcher, and S. H. Glenzer, Free-Electron X-Ray Laser Measurements of Collisional-Damped Plasmons in Isochorically Heated Warm Dense Matter, Phys. Rev. Lett. 115, 115001 (2015).

[104] H. Iyetomi, K. Utsumi, and S. Ichimaru, Dielectric formulation of strongly coupled electron liquids at metallic densities. V. possibility of a charge-density-wave instability, Phys. Rev. B 24, 3226 (1981).

[105] T. Dornheim, T. Sjostrom, S. Tanaka, and J. Vorberger, Strongly coupled electron liquid: Ab initio path integral Monte Carlo simulations and dielectric theories, Phys. Rev. B 101, 045129 (2020).

[106] H. K. Schweng and H. M. Böhm, Finite-temperature electron correlations in the framework of a dynamic local-field correction, Phys. Rev. B 48, 2037 (1993).
[107] A. W. Overhauser, Spin density waves in an electron gas, Phys. Rev. 128, 1437 (1962).

[108] J. P. Perdew and T. Datta, Charge and spin density waves in jellium, Phys. Status Solidi B 102, 283 (1980).

[109] L. M. Sander, J. H. Rose, and H. B. Shore, Charge-density waves in two- and three-dimensional jellium, Phys. Rev. B 21, 2739 (1980).

[110] T. Dornheim, S. Groth, and M. Bonitz, Ab initio results for the static structure factor of the warm dense electron gas, Contrib. Plasma Phys. 57, 468 (2017).

[111] T. Dornheim and J. Vorberger, Overcoming finite-size effects in electronic structure simulations at extreme conditions, J. Chem. Phys. 154, 144103 (2021)

[112] C. Lin, F. H. Zong, and D. M. Ceperley, Twist-averaged boundary conditions in continuum quantum Monte Carlo algorithms, Phys. Rev. E 64, 016702 (2001).

[113] T. Dornheim, Zh. A. Moldabekov, J. Vorberger, and S. Groth, Ab initio path integral Monte Carlo simulation of the uniform electron gas in the high energy density regime, Plasma Phys. Controlled Fusion 62, 075003 (2020).

[114] J. F. Benage, W. R. Shanahan, and M. S. Murillo, Electrical Resistivity Measurements of Hot Dense Aluminum, Phys. Rev. Lett. 83, 2953 (1999).

[115] V. V. Karasiev, L. Calderin, and S. B. Trickey, Importance of finite-temperature exchange correlation for warm dense matter calculations, Phys. Rev. E 93, 063207 (2016).

[116] S. Mazevet, M. P. Desjarlais, L. A. Collins, J. D. Kress, and N. H. Magee, Simulations of the optical properties of warm dense aluminum, Phys. Rev. E 71, 016409 (2005).

[117] M. P. Desjarlais, J. D. Kress, and L. A. Collins, Electrical conductivity for warm, dense aluminum plasmas and liquids, Phys. Rev. E 66, 025401(R) (2002).

[118] A. A. Kugler, Bounds for some equilibrium properties of an electron gas, Phys. Rev. A 1, 1688 (1970).

[119] V. V. Karasiev, S. B. Trickey, and J. W. Dufty, Status of free-energy representations for the homogeneous electron gas, Phys. Rev. B 99, 195134 (2019).

[120] H. Haberland, M. Bonitz, and D. Kremp, Harmonics generation in electron-ion collisions in a short laser pulse, Phys. Rev. E 64, 026405 (2001). 